\title{
Non-adiabatic quantum dynamics without potential energy surfaces based on second-quantized electrons: application within the framework of the MCTDH method
}

\author{
Sudip Sasmal ${ }^{1, \text { a) }}$ and Oriol Vendrell ${ }^{1,2, b)}$ \\ 1) Theoretische Chemie, Physikalisch-Chemisches Institut, Universität Heidelberg, Im Neuneheimer Feld 229, \\ 69120 Heidelberg, Germany \\ ${ }^{2)}$ Interdisciplinary Center for Scientific Computing, Universität Heidelberg, Im Neuneheimer Feld 205 , \\ 69120 Heidelberg, Germany
}

(Dated: 2 October 2020)

A first principles quantum formalism to describe the non-adiabatic dynamics of electrons and nuclei based on a second quantization representation (SQR) of the electronic motion combined with the usual representation of the nuclear coordinates is introduced. This procedure circumvents the introduction of potential energy surfaces and non-adiabatic couplings, providing an alternative to the Born-Oppenheimer approximation. An important feature of the molecular Hamiltonian in the mixed first quantized representation for the nuclei and the SQR representation for the electrons is that all degrees of freedom, nuclear positions and electronic occupations, are distinguishable. This makes the approach compatible with various tensor decomposition ansätze for the propagation of the nuclear-electronic wavefunction. Here, we describe the application of this formalism within the multi-configuration time-dependent Hartree (MCTDH) framework and its multilayer generalization, corresponding to Tucker and hierarchical Tucker tensor decompositions of the wavefunction, respectively. The approach is applied to the calculation of the photodissociation cross-section of the $\mathrm{HeH}^{+}$ molecule under extreme ultraviolet irradiation, which features non-adiabatic effects and quantum interferences between the two possible fragmentation channels, $\mathrm{He}+\mathrm{H}^{+}$and $\mathrm{He}^{+}+\mathrm{H}$. These calculations are compared with the usual description based on ab initio potential energy surfaces and non-adiabatic coupling matrix elements, which fully agree. The proof-of-principle calculations serve to illustrate the advantages and drawbacks of this formalism, which are discussed in detail, as well as possible ways to overcome them. We close with an outlook of possible application domains where the formalism might outperform the usual approach, for example in situations that combine a strong static correlation of the electrons with non-adiabatic electronic-nuclear effects.

\section{INTRODUCTION}

The Born-Oppenheimer approximation $(\mathrm{BOA})^{1,2}$ and its group BOA generalization ${ }^{2-4}$ represent the cornerstone on which the traditional description of the structure of molecules and of the coupled electron-nuclear motion is based. They separate the electronic and nuclear contributions to the molecular wavefunction through the introduction of electronic states parametrized by the nuclear positions, thus resulting in chemical structures and reactions determined by the position and displacements of nuclei over the potential energy surface(s) (PES) provided by the electrons. The BOA is an excellent description of ground-state chemistry but its failure is quite common in the photophysics and photochemistry of molecular systems ${ }^{5,6}$. It breaks down when the energetic gap between the relevant PESs becomes of the order of the spacing between vibrational states, i.e. in the vicinity of avoided crossings and conical intersections where two or more PES interact strongly via vibronic coupling ${ }^{5-10}$. The group BOA (GBOA) is the canonical strategy to deal with these situations ${ }^{3,4}$. A group of electronic states

\footnotetext{
a) e-mail: sudip.sasmal@pci.uni-heidelberg.de

b) e-mail: oriol.vendrell@pci.uni-heidelberg.de
}

assumed uncoupled with the rest is introduced and the non-adiabatic couplings within the group are considered in either adiabatic or diabatic representations, this choice being often a matter of practical convenience. Multiple theoretical methods and strategies exist to both: computing the non-adiabatic couplings (NAC) between electronic states of the group ${ }^{11-14}$ and obtaining a suitable (quasi-)diabatic representation of the coupled nuclear and electronic Hamiltonian ${ }^{8,15-20}$.

Although the GBOA is possibly the most practical strategy to deal with chemical problems in which only a handful of electronic states play a significant role, the calculation of NACs and quasi-diabatic states becomes a formidable task for situations involving a large number of energetically close-lying PES, for example when metal centers $^{21,22}$ and highly excited electronic states are involved $^{23,24}$. These situations arise as well in energy and charge transfer scenarios, where the electronic and nuclear dynamics become inextricable and which are often approached via model Hamiltonians ${ }^{25-29}$. Nonetheless, GBOA non-adiabatic dynamics, both fully quantummechanical and mixed quantum-classical, and involving a large number of electronic states, are still feasible under certain approximations ${ }^{19,24,30}$ and there is much interest in the further development of approaches to obtain NACs in dense electronic manifolds ${ }^{31,32}$. However, it is interesting to consider the fact that the specific electronic state 
occupied by the system at every time during the relevant dynamics is often not an experimental observable that needs to be determined, but rather an auxiliary device along the calculation of, e.g., rates, time-scales and final outcomes of chemical processes.

In this work, we propose an alternative $a b$ initio approach that circumvents the introduction of a group of electronic states and the calculation of the corresponding PES and NACs. It is based on the second quantization representation (SQR) of the electronic subsystem ${ }^{33}$ where the electronic Hamiltonian acts on the Fock space spanned by occupation-number states constructed from a set of properly diabatized single-particle electronic orbitals. In turn, the nuclear coordinates are treated within the usual first quantization framework. In this nuclearSQR (N-SQR) formalism, the non-adiabatic effects are described by the evolution of the nuclear amplitudes coupled to the dynamics of the orbital occupations of the underlying single-particle basis for the electrons. The combination of a first quantized description of vibrational (or phononic) degrees of freedom and a second quantized description of the electrons is not new. For example, the combination of Hubbard's ${ }^{34}$ and Holstein's ${ }^{35}$ models to describe correlated electronic and lattice motions has a history of more than 40 years in the field of solid-state physics $^{36}$. Taking this route to describe the coupled nonadiabatic dynamics of molecular systems from an $a b$ initio perspective has, to the best of knowledge, not been attempted yet.

An important feature of the N-SQR formalism is that all degrees of freedom of the molecular Hamiltonian, nuclear positions and electronic occupations, are distinguishable, based on the fact that in a SQR representation the particles' indistinguishability is determined by the commutation relations of the corresponding creation and annihilation operators, and not by the symmetry of the wavefunction $^{33}$. This feature permits the straightforward application of low-rank tensor approximations to the full nuclear-electronic wavefunction, for example Tucker and hierarchical Tucker decompositions, or matrix-product states, which is not possible if the electronic part of the wavefunction is to be described by a pair-wise antisymmetric tensor ${ }^{37,38}$.

Here, we focus on the representation and the propagation of the N-SQR wavefunction within the framework of the multiconfiguration time-dependent Hartree $\mathrm{MCTDH}^{39-44}$ method and machinery for second quantized Hamiltonians (MCTDH-SQR) ${ }^{45-48}$ (while including first-quantized nuclear degrees of freedom) and note that other methodological alternatives might be suitable as well. Combining first quantized vibrations and second quantized electrons has been successfully achieved by Wang and Thoss within the context of the MCTDH-SQR approach in applications to model Hamiltonians ${ }^{45,46}$. The MCTDH ansatz corresponds to an optimal time-dependent Tucker decomposition of the multi-dimensional wavefunction; its multilayer (ML$\mathrm{MCTDH})^{49-51}$ generalization corresponds to a hierarchi- cal Tucker decomposition and is therefore also N-SQR compatible. Both the MCTDH and ML-MCTDH ansätze do not consider any particular symmetry between the underlying degrees of freedom.

Circumventing the $(\mathrm{G}) \mathrm{BOA}$ to describe the coupled electronic and nuclear dynamics of molecules is a problem of practical and fundamental significance that has been addressed by multiple researchers and from diverse perspectives $^{52-55}$. A non-exhaustive overview of such efforts to follows. One can construct the total multiconfiguration wavefunction using products of nuclear wavefunctions of electronic Slater determinants ${ }^{52,53}$. This corresponds, in short, to extensions of the MCTDH approach for fermions (MCTDH-F) $)^{56-60}$ to a direct product of time-dependent Slater determinants and an orthonormal nuclear functions. A drawback of such approach is that the primitive basis functions of nuclei and electrons are, strictly speaking, independent of each other. This means, the strong but trivial correlation between the positions of the nuclei and electrons due to their Coulomb attraction is carried into the nuclear-electronic wavefunction and needs to be propagated. Haxton and McCurdy resolved this problem for the special case of diatomic molecules by introducing prolate spheroidal coordinates for the electronic wavefunction ${ }^{52}$. Ulusoy and Nest introduced a set of atomic orbitals on "ghost" centers along the internuclear axis of diatomic systems, thus increasing the effective size of the electronic basis but avoiding the reevaluation of matrix elements at each nuclear position ${ }^{53}$. Due to the description of the electrons in an optimal basis of time-dependent orbitals inherited from the variational MCTDH-F framework, these treatments have been successful at describing small molecular systems in intense laser fields ${ }^{52,53,55,59}$. A limitation of such descriptions, also inherited from MCTDH-F, is that the number of configurations of the electronic subsystem increases combinatorially with the number of electrons and electronic single-particle functions, thus hindering their application beyond small molecular systems. Active-space generalizations of the MCTDH-F method can help in mitigating this scaling ${ }^{60}$.

Reiher and co-workers introduced explicitly correlated basis functions between electrons and nuclei; functions that depend on the electronic-nuclear distances. With this treatment they computed vibrationalelectronic eigenstates of small molecules in a pre-BornOppenheimer fashion and without relying on the concept of molecular structures, i.e. without introducing a nuclear framework a priori and thus challenging the traditional concept of molecular structure ${ }^{54}$. Finally, approaches based on an exact, single-product factorization of the nuclear-electronic wavefunction ${ }^{61-63}$ or using conditional electronic wavefunctions ${ }^{64,65}$ also circumvent the BOA. These types of approaches lead to the picture of a nuclear wavefunction evolving on a time-dependent potential whose equation of motion requires knowledge of the nuclear amplitudes. This results in a complex set of coupled equations that have been mostly deployed on 
model Hamiltonians ${ }^{63,65}$, but which have also been the basis of improved mixed quantum-classical approaches to non-adiabatic dynamics ${ }^{66}$. Closing this overview, it is worth mentioning as well that Reiher has recently proposed a method based on quantum dynamics for electrons and nuclei based on matrix product states that bears formal resemblances to N-SQR ${ }^{67}$. Nonetheless, their approach represents the wavefunction of a system consisting of several nuclear degrees of freedom and a discrete set of electronic states, i.e. in the GBOA framework, using the time-dependent density matrix renormalization group (TD-DMRG) ansatz. In this respect, the method is in its aim similar to applications of the ML-MCTDH algorithm to non-adiabatic dynamics situations ${ }^{51,68}$.

The paper is organized as follows. Sections II A and II B shortly review the fundamental aspects of MCTDH and of its application to indistinguishable fermions in a SQR, respectively. Section IIC introduces the N-SQR Hamiltonian whereas Sec. IID discusses practical and implementation details related to using the N-SQR approach in the framework of MCTDH. Section III describes the computational details of the proof-of-concept application to the photodissociation cross section of the $\mathrm{HeH}^{+}$molecule, on which the N-SQR approach is compared to GBOA calculations. Section IV presents and discusses the results and finally a summary and conclusions including an outlook is provided in Sec. V.

\section{THEORY}

\section{A. MCTDH formalism}

In the MCTDH formalism ${ }^{39,40}$, the $f$-dimensional wavefunction $\Psi\left(x_{1}, x_{2}, \ldots, x_{f}, t\right)$ is expanded in an orthonormal basis of time-dependent single particle functions (SPFs)

$$
\Psi\left(x_{1}, \ldots, x_{f}, t\right)=\sum_{j_{1}=1}^{l_{1}} \cdots \sum_{j_{f}=1}^{l_{f}} A_{j_{1} \ldots j_{f}}(t) \prod_{\kappa=1}^{f} \phi_{j_{k}}^{(\kappa)}\left(x_{\kappa}, t\right)=\sum_{J} A_{J}(t) \Phi_{J}(t)
$$

where the usual nomenclature of the MCTDH literature is used ${ }^{41}$ except for the number of SPFs in each DOF $l_{\kappa}$, which is usually labeled by $n_{\kappa}$ and which we reserve for later use. $A_{J}(t)$ is the time-dependent expansion coefficient of the $J$-th configuration marked with multi-index $J$, and $\Phi_{J}(t)$ is the $J$-th time-dependent Hartree product that is formed by a direct product of SPFs for each degree of freedom (DOF). The SPFs $\left(\phi_{j_{\kappa}}^{(\kappa)}\left(x_{\kappa}, t\right)\right)$ are typically represented in a time-independent (primitive) basis

$$
\phi_{j_{\kappa}}^{(\kappa)}\left(x_{\kappa}, t\right)=\sum_{i_{\kappa}}^{N_{\kappa}} c_{i_{\kappa}, j_{\kappa}}^{(\kappa)}(t) \chi_{i_{\kappa}}^{(\kappa)}\left(x_{\kappa}\right)
$$

where $c_{i_{\kappa}}^{(\kappa)} j_{\kappa}(t)$ is a time-dependent expansion coefficient of the $j$-th SPF of the $\kappa$-th DOF. For convenience, these primitive basis functions $\left(\chi_{i_{\kappa}}^{(\kappa)}\left(x_{\kappa}\right)\right)$ are often chosen as a discrete variable representation (DVR), thus greatly simplifying the evaluation of the matrix elements of the potential energy operator ${ }^{41}$.

Therefore, one can view the MCTDH ansatz as a two layer wavefunction where, in the bottom layer, the $c_{i_{\kappa}, j_{\kappa}}^{(\kappa)}(t)$ coefficients represent optimally evolving timedependent SPFs along each coordinate, whereas the upper layer of $A_{J}(t)$ coefficients represents the total multidimensional wavefunction in the direct products basis of the time-dependent SPFs. In MCTDH, one often combines the $f$ physical coordinates $\left(x_{1}, x_{2}, \ldots, x_{f}\right)$ into $d$ groups of logical multidimensional coordinates $\left(q_{1}, q_{2}, \ldots, q_{d}\right)^{42}$. This has no effect in the equations of motion (EOM) but is an important element to balance the cost of the propagation between the A-vector and the SPFs.

Finally, the EOM for the MCTDH ansatz are obtained using the time dependent Dirac-Frenkel variational principle $^{69}$

$$
\left\langle\partial \Psi\left|H-i \frac{\partial}{\partial t}\right| \Psi\right\rangle=0 .
$$

With the constrains $\left\langle\phi_{j}^{(\kappa)} \mid \dot{\phi}_{i}^{(\kappa)}\right\rangle=0$, the EOM for the MCTDH ansatz read ${ }^{41}$

$$
\begin{aligned}
i \dot{A}_{J} & =\sum_{L}\left\langle\Phi_{J}|H| \Phi_{L}\right\rangle \dot{A}_{L} \\
i \dot{\boldsymbol{\phi}}^{(\kappa)} & =\left(\mathbf{1}-\boldsymbol{P}^{(\kappa)}\right)\left(\boldsymbol{\rho}^{(\kappa)}\right)^{-1}\langle\boldsymbol{H}\rangle^{(\kappa)} \boldsymbol{\phi}^{(\kappa)}
\end{aligned}
$$

where the vector notation $\phi^{(\kappa)}=\left(\phi_{1}^{(\kappa)}, \phi_{2}^{(\kappa)}, \ldots, \phi_{l_{\kappa}}^{(\kappa)}\right)^{T}$ is used. $\boldsymbol{P}^{(\kappa)}$ is the projector on the space spanned by the SPFs of the $\kappa$-th DOF and $\boldsymbol{\rho}^{(\kappa)}$ and $\langle\boldsymbol{H}\rangle^{(\kappa)}$ are the density matrix and the mean field of $\kappa$-th $\mathrm{DOF}^{41}$. The efficiency gain in MCTDH compared to the standard method (propagating directly on the primitive basis) arises from the fact that the number optimal of time-dependent configurations that need to be propagated to describe the correlation of the system is usually much smaller than the number of primitive configurations $\left(\prod_{\kappa=1}^{f} l_{\kappa}\right.$ compared to $\prod_{\kappa=1}^{f} N_{\kappa}$ ). It is, of course, possible to identify counterexamples for very highly correlated systems ${ }^{70}$. In such cases, the number of timedependent configurations needed to achieve convergence 
with MCTDH approaches the number of primitive configurations and the overhead of the algorithm renders it less efficient. Mode combination ${ }^{42}$ and especially the multilayer (ML) approach ${ }^{49-51,71}$, which is implemented in the Heidelberg MCTDH package ${ }^{51,72}$, can boost the efficiency further. This has allowed to describe systems with hundreds and up to thousands of DOF for some model Hamiltonians ${ }^{51,73-75}$.

In the MCTDH formalism introduced above, the coordinates $x_{\kappa}$ correspond to distinguishable DOF. Hence, the ansatz can be regarded as a tensor contraction in Tucker format

$$
C_{i_{1}, \ldots, i_{f}}(t)=\sum_{j_{1}, \ldots, j_{f}}^{l_{1}, \ldots, l_{f}} A_{j_{1}, \ldots, j_{f}}(t) \prod_{\kappa=1}^{f} c_{i_{\kappa}, j_{\kappa}}^{(\kappa)}(t)
$$

of the expansion coefficients of the primitive basis functions $C_{i_{1}, \ldots, i_{f}}(t)$. The coefficients $c_{i_{\kappa}, j_{\kappa}}^{(\kappa) j}(t)$ can be arranged as $l_{\kappa} \times N_{\kappa}$ matrices while the $A$-vector is the core tensor of the contraction. Hence, its rank is smaller than the rank of the exact (within the primitive basis) $C$ vector. In mode combination ${ }^{41}$, the mode tensors $c_{i_{\kappa}, j_{\kappa}}^{(\kappa)}$ are chosen of a larger order than two by turning the $i_{\kappa}$ index into a multi-index. Correspondingly, the core tensor rank is further reduced. Once the mode tensors have too many terms, a Tucker decomposition can be applied to them, which results in the hierarchical Tucker format of multilayer-(ML-)MCTDH. The key requirement for this hierachical construct to be possible is that the primitive indices $\left\{i_{1}, \ldots, i_{f}\right\}$ refer to a direct product basis of distinguishable DOF.

It is still possible, although inefficient, to describe indistinguishable DOF within the original MCTDH (not ML) framework. For this, the SPFs must be restricted to one single set for all particles and the propagation must be started with an (anti)symmetric $A$-vector. Early applications of MCTDH to the field of cold Bosons relied on this strategy ${ }^{76,77}$. Clearly, this case is not amenable to a hierarchical Tucker decomposition because the entries of the $A$-vector in Eq. (6) are related by symmetry upon permutation of two indices: the grouping of particle DOFs in logical coordinates is not possible. However, the original MCTDH theory has been extended by introducing permanents for Bosons ${ }^{57,78}$ and Slater determinants for Fermions ${ }^{5-60}$, which avoids the presence of redundant (exchange symmetry-related) A-vector entries in the wavefunction. These approaches are made efficient by using standard rules to calculate the matrix elements of the (anti-)symmetric configurations, e.g. Slater-Condon rules for Fermions.

\section{B. MCTDH-SQR}

\section{General aspects}

A fundamentally different alternative to describe systems of indistinguishable particles is to use a second quantization representation (SQR). This approach was described and applied for the first time by Thoss and Wang in the context of MCTDH and termed MCTDH in SQR (MCTDH-SQR) ${ }^{45}$. The underlying idea is quite general and widely used besides the MCTDH context. Here we describe the general features of this formulation for the sake of completeness before describing the particular aspects of our implementation.

In SQR, the state of the system can be described in occupation number formalism as a superposition of Fock states

$$
|\boldsymbol{n}\rangle=\left|n_{1}, n_{2}, \ldots, n_{M}\right\rangle,
$$

where $n_{\kappa}$ represents the occupation of the $\kappa$-th single particle state (SPS, to be differentiated from the MCTDH SPF introduced above) and $M$ corresponds to the total number of SPS. $n_{\kappa}$ can be equal to 0 or any positive integer for Bosons and either 0 or 1 for Fermions. The indices of the primitive tensor of coefficients in

$$
|\Psi(t)\rangle=\sum_{n_{1}, \ldots, n_{M}} C_{n_{1}, \ldots, n_{M}}(t)\left|n_{1}, n_{2}, \ldots, n_{M}\right\rangle
$$

refer now to an underlying set of distinguishable degrees of freedom, the occupations of each SPS of the manybody system. These Fock space states can be written as well as the direct product of the occupation number states of the $M$ Fock subspaces, one for each SPS

$$
|\boldsymbol{n}\rangle=\left|n_{1}\right\rangle \otimes\left|n_{2}\right\rangle \otimes \cdots \otimes\left|n_{M}\right\rangle,
$$

where we simply are acknowledging the fact that the coefficients in the wavefunction (8) can be regarded as an $M$-dimensional tensor. Because of the distinguishability of the degrees of freedom, a hierarchical Tucker decomposition of the primitive tensor is possible and therefore the original MCTDH formalism (or its multilayer generalisation) is, in principle, applicable without further modification.

What remains to be discussed is how the Hamiltonian of the many-body system acts on the wavefunction, and this is different for bosons and for fermions. MCTDH is most efficient when the operator takes the form of a sum of product terms, each acting on a degree of freedom. The SQR operator for a many-body system of bosons or fermions (with up to two-body interactions) reads

$$
\hat{H}=\sum_{i j} h_{i j} \hat{a}_{i}^{\dagger} \hat{a}_{j}+\frac{1}{2} \sum_{i j k l} v_{i j k l} \hat{a}_{i}^{\dagger} \hat{a}_{j}^{\dagger} \hat{a}_{l} \hat{a}_{k},
$$

where the $\hat{a}_{i}^{(\dagger)}$ correspond to the annihilation (creation) operators fulfilling the corresponding commutation relations and

$$
\begin{aligned}
h_{i j} & =\left\langle\phi_{i}(1)\left|-\frac{1}{2} \nabla_{1}^{2}-\sum_{A=1}^{M} \frac{Z_{A}}{r_{1 A}}\right| \phi_{j}(1)\right\rangle \\
v_{i j k l} & =\left\langle\phi_{i}(1) \phi_{j}(2)|v(1,2)| \phi_{k}(1) \phi_{l}(2)\right\rangle
\end{aligned}
$$

are the one- and two-body integrals, respectively, involving the SPSs $\phi_{i}(1)$. Although our primary interest is 
in the fermionic (electronic) case, we describe first the treatment of bosons, which is conceptually simpler.

The creation and annihilation operators for bosons fulfill the commutation relations

$$
\begin{aligned}
{\left[\hat{a}_{i}, \hat{a}_{j}^{\dagger}\right] } & =\hat{a}_{i} \hat{a}_{j}^{\dagger}-\hat{a}_{j}^{\dagger} \hat{a}_{i}=\delta_{i j} \\
{\left[\hat{a}_{i}^{\dagger}, \hat{a}_{j}^{\dagger}\right] } & =\left[\hat{a}_{i}, \hat{a}_{j}\right]=0,
\end{aligned}
$$

which imply that the creation or annihilation of a particle in a specific SPS does not carry with it a multiplicative phase factor depending on the occupation of the other SPSs $^{33}$. Additionally, for $j=i$ the relations $(13,14)$ coincide with the ladder operators of a harmonic oscillator. Thus, as is well known, the Hamiltonian of the many-body bosonic system is isomorph with the Hamiltonian of a set of coupled harmonic oscillators, where each of them represents the particle occupation of a SPS. Hence, the Hamiltonian (10) is, for the bosonic case, of product form. In practical applications, e.g. within MCTDH, one can either directly use the matrix representation of the ladder (creation/annihilation) operators, or introduce their standard form as a function of position and momentum operators. Multiple applications of the MCTDH method are found in the literature, where coupled oscillators are involved. In particular, the ML-MCTDH approach has been used to describe about 1500 coupled oscillators in the Henon-Heiles model ${ }^{51}$, and other works considering even larger numbers of degrees of freedom can be found ${ }^{73,74}$.

For the case of fermions, the commutation relations are

$$
\begin{array}{r}
\left\{\hat{a}_{i}, \hat{a}_{j}^{\dagger}\right\}=\hat{a}_{i} \hat{a}_{j}^{\dagger}+\hat{a}_{j}^{\dagger} \hat{a}_{i}=\delta_{i j} \\
\left\{\hat{a}_{i}^{\dagger}, \hat{a}_{j}^{\dagger}\right\}=\left\{\hat{a}_{i}, \hat{a}_{j}\right\}=0 .
\end{array}
$$

These have as a consequence that the operator $\hat{a}_{s}$ acting on a Fock-space ket

$$
\begin{array}{r}
\hat{a}_{s}\left|n_{1}, n_{2}, \ldots, n_{M}\right\rangle=\hat{a}_{s}\left(\hat{a}_{1}^{\dagger}\right)^{n_{1}}\left(\hat{a}_{2}^{\dagger}\right)^{n_{2}} \cdots\left(\hat{a}_{M}^{\dagger}\right)^{n_{M}}|\mathrm{vac}\rangle \\
=(-1)^{S_{s}}\left(\hat{a}_{1}^{\dagger}\right)^{n_{1}}\left(\hat{a}_{2}^{\dagger}\right)^{n_{2}} \cdots\left(\hat{a}_{s} \hat{a}_{s}^{\dagger}\right) \cdots\left(\hat{a}_{M}^{\dagger}\right)^{n_{M}}|\mathrm{vac}\rangle
\end{array}
$$

accumulates a phase factor $S_{s}=\sum_{k=1}^{s-1} n_{k}$ that depends on the occupation of all SPS (spin-orbitals for the electronic case) before the $s$-th position, and the same is true for $\hat{a}_{s}^{\dagger 33}$. This phase complicates the application of Hamiltonian (10) to the wavefunction. Clearly, the operators $\hat{a}_{s}^{(\dagger)}$ operate beyond their index $s$ and therefore are, in general, not of product form with respect to the primitive degrees of freedom or combined modes used in the MCTDH Ansatz.

One possible solution, the one we adopt here and also the one proposed by Wang and Thoss ${ }^{45}$, is to map the fermionic SQR Hamiltonian onto an equivalent spin Hamiltonian. Formally, this is done by mapping fermionic DOF onto spin DOF using the (inverse) Jor-
dan-Wigner transformation ${ }^{79}$, according to . i.e.,

$$
\begin{aligned}
& \hat{a}_{i}^{\dagger}=\exp \left(+i \pi \sum_{k=1}^{i-1} \hat{\sigma}_{k}^{+} \hat{\sigma}_{k}^{-}\right) \hat{\sigma}_{i}^{+} \\
& \hat{a}_{i}=\exp \left(-i \pi \sum_{k=1}^{i-1} \hat{\sigma}_{k}^{+} \hat{\sigma}_{k}^{-}\right) \hat{\sigma}_{i}^{-}
\end{aligned}
$$

and

$$
\hat{\sigma}_{k}^{+} \hat{\sigma}_{k}^{-}=\hat{n}_{k}=\frac{1}{2}\left(\hat{\sigma}_{k}^{z}+1\right)
$$

where $\hat{\sigma}_{i}^{+}=\frac{1}{2}\left(\hat{\sigma}_{i}^{x}+i \hat{\sigma}_{i}^{y}\right), \hat{\sigma}_{i}^{-}=\frac{1}{2}\left(\hat{\sigma}_{i}^{x}-i \hat{\sigma}_{i}^{y}\right)$, and $\hat{\sigma}_{k}^{z}$ are the standard spin ladder operators with Pauli matrices $\boldsymbol{\sigma}^{x}, \boldsymbol{\sigma}^{y}$ and $\boldsymbol{\sigma}^{z}$. Following this prescription, the creation and annihilation operators (Eq. 18) can be rewritten as ${ }^{45}$

$$
\begin{aligned}
& \hat{a}_{i}^{\dagger}=\prod_{k=1}^{i-1}(-1)^{n_{k}} \cdot \hat{\sigma}_{i}^{+}=\prod_{k=1}^{i-1} \hat{S}_{k} \cdot \hat{\sigma}_{i}^{+} \\
& \hat{a}_{i}=\prod_{k=1}^{i-1}(-1)^{n_{k}} \cdot \hat{\sigma}_{i}^{-}=\prod_{k=1}^{i-1} \hat{S}_{k} \cdot \hat{\sigma}_{i}^{-}
\end{aligned}
$$

where $\hat{S}_{k}$ are the sign change operators acting locally on index $k$ and their matrix representation reads

$$
\begin{aligned}
\boldsymbol{\sigma}^{+}=\left(\begin{array}{cc}
0 & 0 \\
1 & 0
\end{array}\right) ; \quad \boldsymbol{\sigma}^{-}=\left(\begin{array}{ll}
0 & 1 \\
0 & 0
\end{array}\right) ; \\
\boldsymbol{S}=\left(\begin{array}{cc}
1 & 0 \\
0 & -1
\end{array}\right) ; \quad \boldsymbol{n}=\left(\begin{array}{ll}
0 & 0 \\
0 & 1
\end{array}\right) .
\end{aligned}
$$

The equivalent form of the second quantized electronic Hamiltonian in product form is given by

$$
\begin{aligned}
\hat{H}_{e}= & \sum_{i j} h_{i j}\left(\prod_{q=a+1}^{b-1} \hat{S}_{q}\right) \hat{\sigma}_{i}^{+} \hat{\sigma}_{j}^{-} \\
+ & \frac{1}{2} \sum_{i j k l} v_{i j k l}\left(\prod_{q=a+1}^{b-1} \hat{S}_{q} \prod_{q^{\prime}=c+1}^{d-1} \hat{S}_{q^{\prime}}\right) \\
& \operatorname{sgn}(j-i) \operatorname{sgn}(l-k) \hat{\sigma}_{i}^{+} \hat{\sigma}_{j}^{+} \hat{\sigma}_{l}^{-} \hat{\sigma}_{k}^{-} .
\end{aligned}
$$

Here the indices $(a, b, c, d)$ correspond to the $(i, j, k, l)$ indices, but ordered from smaller to larger and the sgn function is defined as

$$
\operatorname{sgn}(x)= \begin{cases}1, & \text { if } x \geq 0 \\ -1, & \text { otherwise }\end{cases}
$$

\section{Representations of the SQR wavefunction and operator}

There are two limiting wavefunction ansätze when applying the MCTDH-SQR formalism to fermionic systems: (i) Each spin degree of freedom (S-DOF) is described by one SPF. The second quantized wavefunction is then described by a single Hartree product. (ii) Each S-DOF 

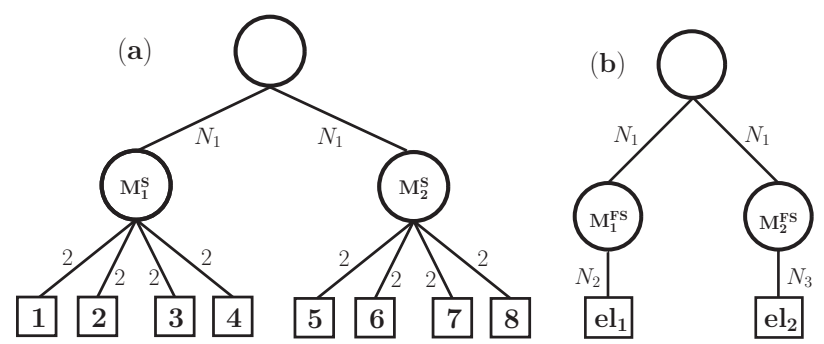

FIG. 1. Tree structures for the MCTDH electronic wavefunction containing 8 spin orbitals. (a) MCTDH wavefunction tree, in which spin orbitals are considered as the primitive electronic DOFs (S-DOF). (b) MCTDH wavefunction tree, in which the Fock space of the 4 combined spin orbitals is considered as a single electronic DOF (FS-DOF).

is described by two SPFs. In this case the wavefunction has $2^{M}$ configurations, is exact, and spans all states of the underlying Fock space. Limiting case (i) is a poor representation of correlated states, whereas case (ii) becomes quickly unaffordable as the number of spin-orbitals increases. Therefore, the only practical alternative to apply MCTDH-SQR to fermions is to group primitive spin degrees of freedom together, either through mode combination or through the generalization to ML-MCTDHSQR. Grouping two or more S-DOFs the total Fock space becomes

$$
\mathcal{F}(M)=f_{1}\left(m_{1}\right) \otimes f_{2}\left(m_{2}\right) \otimes \cdots \otimes f_{d}\left(m_{d}\right)
$$

where $f_{\kappa}\left(m_{\kappa}\right)$ denotes the sub-Fock space of $m_{\kappa}$ S-DOFs with $2^{m_{\kappa}}$ Fock states and

$$
M=\sum_{\kappa=1}^{d} m_{\kappa} .
$$

In MCTDH language, $f_{\kappa}\left(m_{\kappa}\right)$ corresponds to the primitive space of the $\kappa$-th logical coordinate.

One can go one step further and represent the states of the sub-Fock space $f_{\kappa}\left(m_{\kappa}\right)$ as a new primitive degree of freedom. We refer to this representation of the primitive degrees of freedom as Fock space DOF (FS-DOF). There are several advantages when using FS-DOFs over S-DOFs:

1. The Fock space of each FS-DOFs can be statically pruned before the calculation. This static pruning is not possible in the S-DOF representation. The pruning of the Fock space is equivalent to removing unwanted grid points from a multidimensional grid in a discrete variable representation (DVR) ${ }^{80}$. A similar strategy, termed adiabatic contraction, was used by Wang and Thoss in their applications using the ML-MCTDH-SQR method ${ }^{81,82}$. The criteria to prune the primitive space are different though. Whereas they employ an energetic cutoff, we consider what are the possible electronic occupations of the subspace according to various criteria. As an example, let us consider a system containing 8 spin orbitals that are divided into two groups, each with 4 spin orbitals. The MCTDH wavefunction trees for S-DOF and FS-DOF are given in Fig. 1. For S-DOFs, each DOF has two primitive states $|0\rangle$ and $|1\rangle$ and the Fock space spanned by each combined mode $M_{1}^{F S}$ and $M_{2}^{F S}$ mode, Fig. 1(a), is

$$
\begin{gathered}
\mathcal{F}(4) \ni(|0\rangle \oplus|1\rangle)_{1} \otimes(|0\rangle \oplus|1\rangle)_{2} \otimes \\
(|0\rangle \oplus|1\rangle)_{3} \otimes(|0\rangle \oplus|1\rangle)_{4}
\end{gathered}
$$

with $2^{4}=16$ Fock states. In the FS-DOF case, each of the $M_{1}^{F S}$ and $M_{2}^{F S}$ degrees of freedom, Fig. 1(b), is spanned, in principle, by the 16 original Fock states. In practice, we one can prune the FS-DOF by either allowing only a certain range of electronic occupations in each FS-DOF, by further removing certain occupation number states that are considered chemically irrelevant (for example based on energetic arguments), or both. For the above mentioned example, if the spin orbitals are energetically ordered with respect to a pre-existing first quantization mean-field calculation, then in the lowest energy configuration of the mean-field all the orbitals in $e l_{1}$ are occupied $(|1,1,1,1\rangle)$ and all orbitals in $e l_{2}$ are empty $(|0,0,0,0\rangle)$. Now, if one decides to allow only upto two holes in $e l_{1}$, the occupation number states of the $\mathcal{H}(4,0)$ and $\mathcal{H}(4,1)$ Hilbert spaces are removed from the corresponding $\mathcal{F}(4)$ Fock space, which shrinks from 16 to 9 occupation number states. Equivalently, one would in this case also limit the maximum number of electrons in the $e l_{2}$ Fock space to two, thus eliminating from it the occupation number states of the $\mathcal{H}(4,3)$ and $\mathcal{H}(4,4)$ Hilbert spaces.

2. The operators acting on FS-DOFs are very sparse and correspond to pure mappings. This is probably the most important reason for introducing FSDOFs and the one that can lead to the largest efficiency gains in computations. To illustrate this, let us consider again a simple example where the $(2 \times 2)$ matrices

$$
\boldsymbol{\sigma}_{(1)}^{+} \boldsymbol{S}_{(2)} \boldsymbol{S}_{(3)}=\left(\begin{array}{ll}
0 & 0 \\
1 & 0
\end{array}\right)_{(1)}\left(\begin{array}{cc}
1 & 0 \\
0 & -1
\end{array}\right)_{(2)}\left(\begin{array}{cc}
1 & 0 \\
0 & -1
\end{array}\right)_{(3)}
$$

are applied to a sequence of three combined spin degrees of freedom. The $(8 \times 8)$ matrix performing the same operation on the corresponding FS-DOFs reads

$$
\left(\begin{array}{cccccccc}
0 & 0 & 0 & 0 & 0 & 0 & 0 & 0 \\
0 & 0 & 0 & 0 & 0 & 0 & 0 & 0 \\
0 & 0 & 0 & 0 & 0 & 0 & 0 & 0 \\
0 & 0 & 0 & 0 & 0 & 0 & 0 & 0 \\
1 & 0 & 0 & 0 & 0 & 0 & 0 & 0 \\
0 & -1 & 0 & 0 & 0 & 0 & 0 & 0 \\
0 & 0 & -1 & 0 & 0 & 0 & 0 & 0 \\
0 & 0 & 0 & 1 & 0 & 0 & 0 & 0
\end{array}\right) .
$$


All such combined matrix operators have the following properties: there is at most one entry different than 0 in each row and column, which can be either equal to 1 or -1 . In an $n \times n$ matrix, at most $n$ entries are different than 0 . These properties can be used to greatly increase the efficiency of the multiplication of these matrices to vectors of coefficients (representing an MCTDH single particle function). These operations can be performed by dedicated subroutines by mapping coefficients and avoiding actual number multiplications and summations. Note, for example, that the combination of 10 spin-orbitals into one FS-DOF leading to a Fock space with 1024 occupation number states results in matrix operators with at most $\approx 1 / 1000$ non-zero entries, and even larger FS-DOFs are possible.

3. The pruning of the FS-DOF spaces can result in fewer product terms of the second quantized Hamiltonian (22). This is the case for product terms, e.g. of the form of Eq. (27), that in the original sub-Fock space link configurations that in the FS-DOF have been pruned.

\section{Non-adiabatic quantum dynamics in second quantization}

One of the main purposes of this paper is to introduce and describe the treatment of the coupled nuclear and electronic dynamics based on an SQR representation of the electronic subsystem. The full non-relativistic molecular Hamiltonian is given by

$$
\hat{H}=\hat{T}_{n}+\hat{W}_{n}+\hat{H}_{e}
$$

where the nuclear kinetic energy $\left(\hat{T}_{n}\right)$ and the nuclearnuclear potential energy $\left(\hat{W}_{n}\right)$ operators are given by

$$
\hat{T}_{n}=\sum_{\alpha=1}^{N}-\frac{\hbar^{2}}{2 m_{\alpha}} \vec{\nabla}_{\alpha}^{2}
$$

and

$$
\hat{W}_{n}=\sum_{\alpha, \beta=1}^{N} \frac{Q_{\alpha} Q_{\beta} e^{2}}{\left|\vec{R}_{\alpha}-\vec{R}_{\beta}\right|}
$$

respectively. Here, the indices $\alpha, \beta$ run over all nuclear DOFs. In this section it will be convenient to express the electronic part $\left(\hat{H}_{e}\right)$ of the total molecular Hamiltonian in terms of second-quantized fermionic field operators $\hat{\psi}^{\dagger}(\vec{x})$ and $\hat{\psi}(\vec{x})$

$$
\begin{aligned}
\hat{H}_{e} & =\int d r_{1}^{3} \hat{\psi}^{\dagger}\left(\overrightarrow{x_{1}}\right)\left(-\frac{\hbar^{2}}{2 m_{e}} \vec{\nabla}^{2}+\sum_{\alpha=1}^{N} \frac{-Q_{\alpha} e^{2}}{\left|\overrightarrow{R_{\alpha}}-\vec{r}_{1}\right|}\right) \hat{\psi}\left(\overrightarrow{x_{1}}\right) \\
& +\int d r_{1}^{3} \int d r_{2}^{3} \hat{\psi}^{\dagger}\left(\overrightarrow{x_{1}}\right) \hat{\psi}^{\dagger}\left(\overrightarrow{x_{2}}\right) \frac{e^{2}}{\left|\overrightarrow{r_{1}}-\overrightarrow{r_{2}}\right|} \hat{\psi}\left(\overrightarrow{x_{2}}\right) \hat{\psi}\left(\overrightarrow{x_{1}}\right),
\end{aligned}
$$

where $\vec{x}_{j}$ denotes the spatial and spin coordinates of the fermionic particles. The simplest situation corresponds to the case in which the field operators are expanded in a basis of electronic spin-orbitals that are independent of the nuclear positions

$$
\begin{aligned}
\hat{\psi}^{\dagger}(\vec{x}) & =\sum_{j=1}^{F} \chi_{j}^{*}(\vec{x}) \hat{a}_{j}^{\dagger} \\
\hat{\psi}(\vec{x}) & =\sum_{j=1}^{F} \chi_{j}(\vec{x}) \hat{a}_{j} .
\end{aligned}
$$

Hamiltonian (29-32) can be re-expressed using the mode expansions $(33,34)$ resulting in

$$
\hat{H}=\hat{T}_{n}+\hat{W}_{n}+\sum_{i j}^{F} h_{i j}(\mathbf{R}) \hat{a}_{i}^{\dagger} \hat{a}_{j}+\frac{1}{2} \sum_{i j k l}^{F} v_{i j k l} \hat{a}_{i}^{\dagger} \hat{a}_{j}^{\dagger} \hat{a}_{l} \hat{a}_{k},
$$

where

$$
h_{i j}(\mathbf{R})=\left\langle\chi_{i}\left(\vec{x}_{1}\right)\left|\frac{-\vec{\nabla}^{2}}{2}+\sum_{\alpha=1}^{N} \frac{-Q_{\alpha}}{\left|\vec{R}_{\alpha}-\vec{r}_{1}\right|}\right| \chi_{j}\left(\vec{x}_{1}\right)\right\rangle
$$

and

$$
v_{i j k l}=\left\langle\chi_{i}\left(\vec{x}_{1}\right) \chi_{j}\left(\vec{x}_{2}\right)\left|\frac{1}{\left|\vec{r}_{1}-\vec{r}_{2}\right|}\right| \chi_{k}\left(\vec{x}_{1}\right) \chi_{l}\left(\vec{x}_{2}\right)\right\rangle
$$

are the one- and two-body integrals of the electronic subsystem, respectively, and the coupling of the electrons and nuclei occurs only through the one-body electronic term.

In applications to molecular systems it may be more convenient to represent the electronic problem in a single particle basis that depends on the nuclear positions, thus removing to a large extent the artificial correlations among the positions of the nuclei and electrons in space and thus keeping the number of electronic spin-orbitals as small as possible. In such a basis, the same field operators $(33,34)$ are expanded as

$$
\begin{aligned}
\hat{\psi}^{\dagger}(\vec{x}) & =\sum_{j=1}^{F} \varphi_{j}^{*}(\vec{x} ; \mathbf{R}) \hat{b}_{j}^{\dagger}(\mathbf{R}) \\
\hat{\psi}(\vec{x}) & =\sum_{j=1}^{F} \varphi_{j}(\vec{x} ; \mathbf{R}) \hat{b}_{j}(\mathbf{R}) .
\end{aligned}
$$


At first sight it may look now as if one will obtain again Hamiltonian (35), but now expressed in the nucleardependent basis. This is not the case because the occupation number states carry an implicit nuclear-positon dependency (cf. Eq. (43)) that results in non-adiababtic coupling (NAC) terms of kinetic nature:

$$
\begin{aligned}
\left\langle\{\vec{m}\}\left|\hat{T}_{n}\right|\{\vec{n}\}\right\rangle & =\hat{T}_{n} \delta_{\vec{m} \vec{n}} \\
& -\frac{1}{M}\langle\{\vec{m}\}|\nabla|\{\vec{n}\}\rangle \nabla \\
& -\frac{1}{2 M}\left\langle\{\vec{m}\}\left|\nabla^{2}\right|\{\vec{n}\}\right\rangle .
\end{aligned}
$$

To evaluate the SQR NACs we first combine Eqs. (33, $34)$ and $(38,38)$ and express the the position-dependent creation (annihilation) operators, in terms of the position-independent operators as

$$
\begin{aligned}
\hat{b}_{j}(\mathbf{R}) & =\sum_{k=1}^{F}\left\langle\varphi_{j}(\mathbf{R}) \mid \chi_{k}\right\rangle \hat{a}_{k} \\
\hat{b}_{j}^{\dagger}(\mathbf{R}) & =\sum_{j=1}^{F}\left\langle\chi_{k} \mid \varphi_{j}(\mathbf{R})\right\rangle \hat{a}_{k}^{\dagger},
\end{aligned}
$$

respectively, and then express the occupation number representation kets starting from the vacuum state as

$$
|\{\vec{n}\}\rangle=\prod_{k=1}^{F}\left(\hat{b}_{k}^{\dagger}\right)^{n_{k}}|0\rangle
$$

Here and in the following we drop the $R$-dependence of the $\hat{b}_{k}$ and $\hat{b}_{k}^{\dagger}$ for notational simplicity. Note that $\hat{b}_{k}$ and $\hat{b}_{k}^{\dagger}$ act on the Fock space basis similarly as $\hat{a}_{k}$ and $\hat{a}_{k}^{\dagger}$, and in practice they are replaced by the spin matrices in Eqs.(20,21). Making use of the expressions above one arrives at

$$
\begin{aligned}
\langle\{\vec{m}\}|\nabla|\{\vec{n}\}\rangle & =\left\langle\{\vec{m}\}\left|\nabla \prod_{l=1}^{F}\left(\hat{b}_{l}^{\dagger}\right)^{n_{l}}\right| 0\right\rangle \\
& =\left\langle\{\vec{m}\}\left|\sum_{l} n_{l}\left[\prod_{k<l}\left(\hat{b}_{k}^{\dagger}\right)^{n_{k}}\right]\left[\sum_{q}\left\langle\chi_{q}|\nabla| \varphi_{l}(\mathbf{R})\right\rangle \hat{a}_{q}^{\dagger}\right]\left[\prod_{k>l}\left(\hat{b}_{k}^{\dagger}\right)^{n_{k}}\right]\right| 0\right\rangle \\
& =\left\langle\{\vec{m}\}\left|\sum_{r l} n_{l}\left[\prod_{k<l}\left(\hat{b}_{k}^{\dagger}\right)^{n_{k}}\right]\left[\sum_{q}\left\langle\chi_{q} \mid \varphi_{r}(\mathbf{R})\right\rangle\left\langle\varphi_{r}(\mathbf{R})|\nabla| \varphi_{l}(\mathbf{R})\right\rangle \hat{a}_{q}^{\dagger}\right]\left[\prod_{k>l}\left(\hat{b}_{k}^{\dagger}\right)^{n_{k}}\right]\right| 0\right\rangle \\
& =\left\langle\{\vec{m}\}\left|\sum_{r l} n_{l}\left[\prod_{k<l}\left(\hat{b}_{k}^{\dagger}\right)^{n_{k}}\right]\left[\left\langle\varphi_{r}(\mathbf{R})|\nabla| \varphi_{l}(\mathbf{R})\right\rangle \hat{b}_{r}^{\dagger}\right]\left[\prod_{k>l}\left(\hat{b}_{k}^{\dagger}\right)^{n_{k}}\right]\right| 0\right\rangle,
\end{aligned}
$$

where the nuclear derivative operator acts now on the basis of position dependent spin-orbitals. Finally, the multiplicative $n_{l}$ term in the last line of Eq. (44) can be substituted by $b_{l}\left(b_{l}^{\dagger}\right)^{n_{l}}$, leaving the matrix element unaltered and allowing to re-introduce the ket $|\{\vec{n}\}\rangle$ on the right side

$$
\langle\{\vec{m}\}|\nabla|\{\vec{n}\}\rangle=\sum_{r l}\left\langle\varphi_{r}(\mathbf{R})|\nabla| \varphi_{l}(\mathbf{R})\right\rangle\left\langle\{\vec{m}\}\left|\hat{b}_{r}^{\dagger} \hat{b}_{l}\right|\{\vec{n}\}\right\rangle .
$$

The second order non-adiabatic coupling term is obtained in the same manner and reads

$$
\left\langle\{\vec{m}\}\left|\nabla^{2}\right|\{\vec{n}\}\right\rangle=\sum_{r l}\left\langle\varphi_{r}(\mathbf{R})\left|\nabla^{2}\right| \varphi_{l}(\mathbf{R})\right\rangle\left\langle\{\vec{m}\}\left|\hat{b}_{r}^{\dagger} \hat{b}_{l}\right|\{\vec{n}\}\right\rangle
$$

Using the matrix elements in Eqs. $(45,46)$, the total molecular Hamiltonian (29) takes the form

$$
\hat{H}=\hat{T}_{n}+\hat{W}_{n}+\sum_{p q}^{F}\left[h_{p q}(\mathbf{R})-\frac{1}{M} \mathbf{D}_{p q}(\mathbf{R}) \cdot \nabla-\frac{1}{2 M} G_{p q}(\mathbf{R})\right] \hat{b}_{p}^{\dagger} \hat{b}_{q}+\frac{1}{2} \sum_{p q r s}^{F} v_{p q r s}(\mathbf{R}) \hat{b}_{p}^{\dagger} \hat{b}_{q}^{\dagger} \hat{b}_{s} \hat{b}_{r}
$$

in the basis of position-dependent spin-orbitals, where

$$
\mathbf{D}_{p q}=\left\langle\varphi_{p}(\mathbf{R})|\nabla| \varphi_{q}(\mathbf{R})\right\rangle
$$

and

$$
G_{p q}=\left\langle\varphi_{p}(\mathbf{R})\left|\nabla^{2}\right| \varphi_{q}(\mathbf{R})\right\rangle
$$


represent the first and second order non-adiabatic couplings that arise from the nuclear position dependence of the electronic orbital basis, and the integrals $h_{p q}$ and $v_{p q r s}$ are evaluated in the $\left|\varphi_{q}(\mathbf{R})\right\rangle$ basis.

All degrees of freedom in the molecular Hamiltonian (47), nuclei positions and electronic occupations, are distinguishable. We refer to the combination of a first quantized representation for the nuclei and a second quantized representation of the fermionic subsystem, Hamiltonian (47), as the N-SQR framework. N-SQR enables the application of various forms of tensor decompositions to the corresponding wavefunction, such as hierarchical tensor contractions and matrix product-state representations. Hence, MCTDH and its ML-MCTDH generalization, which can be regarded as a hierarchical Tucker decompositions of the wavefunction, can be readily applied to Hamiltonian Eq. (47) to describe the mixed non-adiabatic dynamics of nuclei and electrons without, in principle, further modification. Nonetheless, practical aspects need to be considered in N-SQR calculations, which are the subject of the next section.

For completeness, Appendix A describes formally the application of the Hamiltonian (47) to a Born-Huang-like expansion of the wavefunction, although this alternative is not used in the actual calculations reported here.

\section{Practical aspects of N-SQR and implementation within MCTDH}

\section{Cutoff strategies for Hamiltonian terms}

A major obstacle in the $a b$ initio description of molecules within the SQR framework is the large number of Hamiltonian terms to be considered, mostly due to the four-index Coulomb integrals $v_{i j k l}$. In this work, we apply two strategies to mitigate this problem:

- Frozen core approximation: - This strategy is widely used in static multi-configuration electronic structure calculations. Low lying molecular orbitals, e.g. linear combinations of atomic $1 s$ orbitals, are energetically well separated from the valence space and, for most practical purposes, remain occupied at all times. Removing them leads to a reduction of the number of degrees of freedom in a similar manner as when introducing reduced dimensionality models in nuclear dynamics, and also to a reduction in the number of Hamiltonian terms. Restricting the lowest energy spin-orbitals to always being occupied and dividing the orbital space into two groups, core orbitals $(i, j, \ldots)$ and active orbitals $(a, b, \ldots)$, the energy of the core region is given by

$$
E^{\text {core }}(\mathbf{R})=\sum_{i} h_{i i}(\mathbf{R})+\frac{1}{2} \sum_{i j}\left(v_{i j i j}(\mathbf{R})-v_{i j j i}(\mathbf{R})(5,50)\right.
$$

which contributes to the Hamiltonian as a potential energy surface for the nuclei. The electrons in the active orbitals $(a, b)$ still interact with the filled core and the one-body transfer integrals $h_{a b}(\mathbf{R})$ need to be adjusted accordingly as

$$
\begin{aligned}
& \tilde{h}_{a b}(\mathbf{R})=h_{a b}(\mathbf{R}) \\
& +\frac{1}{2} \sum_{i}\left(v_{i a i b}(\mathbf{R})+v_{a i b i}(\mathbf{R})-v_{i a b i}(\mathbf{R})-v_{a i i b}(\mathbf{R})\right)
\end{aligned}
$$

A similar strategy can be applied if a group of spinorbitals can be assumed to be, at most, singly occupied at all times. This would be the case, e.g., in single ionization processes, where, per definition, only one electron reaches the orbitals that describe the outgoing particle. In this group of orbitals, only one-body terms of the Hamiltonian need to be retained. Spin-orbitals that are considered to never become populated can be removed from the calculation without further consideration.

- Selective cutoff value for Hamiltonian terms: - It is common in MCTDH calculations to set a cutoff and remove small Hamiltonian terms, e.g., of order $10^{-7}$ or smaller before time propagations are performed. We have observed that this strategy is not so convenient with the electronic SQR Hamiltonian because it consists of thousands of very small contributions which, when removed, lead to noticeable effects. For SQR we proceed in a slightly modified way. We apply a predefined cutoff only to 2-body terms of 3-index and 4-index type, e.g. $v_{1,2,3,3}$ or $v_{1,2,3,4}$. The rationale behind this strategy is that only one- and two-body terms with up to two different indices, the Coulomb and exchange integrals, contribute to the energy of an occupation number state $\left\langle\{\vec{n}\}\left|\hat{H}_{e}\right|\{\vec{n}\}\right\rangle$, so we keep them all. Instead, 3 -index and 4-index integrals participate only in the off-diagonal terms that connect different occupation number states. We have observed that this strategy to be effective in reducing the size of the Hamiltonian in a more or less controllable way. This will be illustrated later in the discussion of the results.

\section{Diabatization of orbitals}

One important aspect of static multi-configuration electronic structure calculations aimed at the generation of potentials and NAC coupling elements is the introduction of a basis of molecular orbitals that is as diabatic as possible $18,20,83-86$. In this case, the NAC couplings between adiabatic electronic states are captured, practically in their entirety, by the configuration interaction (CI) expansion vectors ${ }^{83}$. In the nuclear-SQR framework, the advantage of using (quasi-)diabatic orbitals is similar. The differential terms in Hamiltonian (47) can be neglected and all the non-adiabatic effects are included in the time-evolution of the overall wavefunction.

There are several diabatization procedures described in literature that produce (quasi-)diabatic molecular orbitals ${ }^{18,20,83-86}$, for example imposing conformational uniformity at close-lying geometries ${ }^{18,83,84,86}$ or extracting the orbitals from state-averaged self-consistent field calculations $^{85}$. In this work, we simply reorder the Hartree-Fock (HF) molecular orbitals obtained at each 
nuclear geometry to ensure their continuity. In our selected example this is possible because of two reasons. First, the coupled muclear-electronic dynamics involve a group of highly excited electronic configurations, whereas the electronic ground state is, to a very good approximation, mono-configurational along the whole set of internuclear distances. Hence, the HF mean-field presents no discontinuities. Second, we are considering only one nuclear coordinate. In general, though, obtaining a good set of quasi-diabatic orbitals will be an important aspect of N-SQR calculations, which will need a more detailed consideration in future works.

We need to rearrange the MOs such that each MO retains its character over all geometries. In short, we proceed as follows. We construct an overlap matrix between the MOs of two successive geometries $R, R_{0}$

$$
\begin{aligned}
\mathbf{S}_{\left[R, R_{0}\right]}^{M O} & =\boldsymbol{\Psi}_{[R]}^{T} \boldsymbol{\Psi}_{\left[R_{0}\right]} \\
& =\boldsymbol{C}_{[R]}^{T} \boldsymbol{\Phi}_{[R]}^{T} \boldsymbol{\Phi}_{\left[R_{0}\right]} \boldsymbol{C}_{\left[R_{0}\right]},
\end{aligned}
$$

where the vector notation, e.g. for column vector of MOs $\boldsymbol{\Psi}_{[R]}^{T}=\left(\left|\varphi_{1}\right\rangle,\left|\varphi_{2}\right\rangle, \ldots\right)_{[R]}^{T}$, is used, the subscript denotes the corresponding geometry, and the MOs in terms of atomic orbitals (AOs) are given as

$$
\boldsymbol{\Psi}_{[R]}=\boldsymbol{\Phi}_{[R]} \boldsymbol{C}_{[R]},
$$

where $\boldsymbol{C}_{[R]}$ is the AO-MO transformation matrix with the molecular orbital coefficients in its columns. Since atomic orbitals are, in general, not orthogonal and we are interested in their overlap at different, but close, geometries, a direct multiplication of the coefficient matrices $\boldsymbol{C}_{[R]}$ in Eq. (52) assuming that they flank a unit matrix is not possible. Therefore, we introduce symmetrically orthogonalized atomic orbitals (SO)

$$
\chi_{[R]}=\boldsymbol{\Phi}_{[R]} \boldsymbol{s}_{[R]}^{-\frac{1}{2}},
$$

where $\boldsymbol{s}_{[R]}$ is the overlap matrix between AOs at geometry $R$.

$$
\boldsymbol{s}_{[R]}=\boldsymbol{\Phi}_{[R]}^{T} \boldsymbol{\Phi}_{[R]}
$$

Now, using Eq. (54), we can rewrite the Eq. (52) as

$$
\begin{aligned}
\mathbf{S}_{\left[R, R_{0}\right]}^{M O} & =C_{[R]}^{T} s_{[R]}^{\frac{1}{2}}\left(\chi_{[R]}^{T} \chi_{\left[R_{0}\right]}\right) s_{\left[R_{0}\right]}^{\frac{1}{2}} C_{\left[R_{0}\right]} \\
& \approx C_{[R]}^{T} s_{[R]}^{\frac{1}{2}} s_{\left[R_{0}\right]}^{\frac{1}{2}} C_{\left[R_{0}\right]}
\end{aligned}
$$

where we have approximated the overlap between the SOs of two successive geometries $\left(\chi_{[R]}^{T} \chi_{\left[R_{0}\right]}\right) \approx 1$ as a unit matrix. This is a good approximation as long as the $R$ and $R_{0}$ are close enough. The simple diabatization procedure consists now in rearranging the MOs at geometry $R$ (columns of $\boldsymbol{C}_{[R]}$ ) such that the largest matrix elements of the overlap matrix $\mathbf{S}_{\left[R, R_{0}\right]}^{M O}$ are found in the main diagonal, and in multiply a phase of -1 to the corresponding column of $\boldsymbol{C}_{[R]}$, if needed, to render the diagonal matrix elements positive.

\section{Generation of initial SPFs}

An important aspect in MCTDH calculations is the generation of the initial SPFs in Eq. (2). Recently, Weike and Manthe ${ }^{48}$ showed that if the initial SPFs are eigenfunctions of the local particle-number operator of the corresponding $k$-th sub-Fock space $\hat{n}^{(k)}=\sum_{i_{k}}^{N_{k}} \hat{a}_{i_{k}}^{\dagger} \hat{a}_{i_{k}}$, the MCTDH EOM conserve the particle number of each SPF during the time propagation. This means, the initially occupied $\mathrm{SPF}(\mathrm{s})$ in each sub-Fock space (combined mode or FS-DOF) must correspond to an integer particular particle number. Moreover, the initially unoccupied SPF(s) must also each correspond to an integer particle number and their set has to span various particle numbers such that the mode is flexible enough to represent the evolution of the system in the whole Fock space. Therefore, the choice of both the initially occupied and unoccupied initial SPFs is very important in MCTDHSQR calculations. At the moment, we select the initial SPFs

$$
\left|\phi_{j_{k}}^{(k)}\right\rangle=\sum_{i_{k}}^{N_{k}} c_{i_{k}, j_{k}}^{(k)}\left|\{\vec{n}\}_{i_{k}}\right\rangle
$$

by only mixing primitive occupation-number states that are eigenfunctions of the local particle number operator $\hat{n}^{(k)}\left|\{\vec{n}\}_{i_{k}}\right\rangle=n_{i_{k}}^{(k)}\left|\{\vec{n}\}_{i_{k}}\right\rangle$ with the same number of particles $n_{i_{k}}^{(k)}$.

In molecular problems, the choice of what particlenumbers are spanned by the $k$-th sub-Fock space can be made similarly as when considering restricted active spaces in electronic multi-configuration calculations. For example, one may consider an initial electronic configuration with a set of low energy occupied, and a set of empty (virtual) spin-orbitals, and one may assume that at most $m$ electrons have, at any time, been transferred between the initially occupied and unoccupied spaces. This type of strategy has the added advantage of limiting the primitive particle-number states when using FSDOFs, in contrast to $\mathrm{S}-\mathrm{DOFs}$, where the primitive space is not affected by such considerations.

Since, as pointed out by Manthe ${ }^{48}$, the SPFs conserve the local particle number, and the propagation of the expansion coefficients of the $A$-vector conserves the total particle number, MCTDH-SQR calculations based on SPFs with an integer particle number have entries of the $A$-vector that stay equal to zero at all times. These coefficients refer to configurations of the whole Fock space perpendicular to the initially populated Hilbert space. Currently, our code does not take advantage of this fact, but specialized MCTDH-SQR implementations might take advantage of these considerations.

\section{COMPUTATIONAL DETAILS}

We apply the N-SQR framework in combination with MCTDH-SQR to the photofragmentation of the $\mathrm{HeH}^{+}$ 
molecule upon excitation by XUV photons of about $30 \mathrm{eV}$. Following the photoexcitation process, it is known that non-adiabatic effects couple different fragmentation channels and result in quantum interferences that shape the channel-selected photodissociation cross section $^{87}$. The N-SQR calculations are compared with traditional first quantization Born-Huang ${ }^{2}$ (FQBH) calculations based on the GBOA. We perform the FQBH calculations in the adiabatic representation and thus we use the computed PES and NACs from $a b$ initio quantum chemistry directly and without further transformation to a quasi-diabatic electronic basis. Technical details on the FQBH quantum dynamics calculations in the adiabatic representation are provided in Appendix B.

The uncontracted aug-cc-pVTZ ${ }^{88}$ atomic basis is used for both $\mathrm{H}$ and $\mathrm{He}$ to generate the underlying spinorbitals for the N-SQR calculation. As already mentioned, we use the Hartree-Fock molecular orbitals (MO) as the one-particle basis used to construct the matrix elements in Hamiltonian. (47). The primitive orbital space consists of 10 spin-orbitals (5 lowest-energy spatial MOs of $\Sigma$ symmetry in the $C_{\infty}$ point group) of which two are occupied in the HF reference configuration. The one-electron and two-electron matrix elements in the atomic orbital (AO) basis and the AO-MO transformation coefficients $\boldsymbol{C}_{[R]}$ are obtained using the PSI4 program package ${ }^{89}$. The columns of the $\boldsymbol{C}_{[R]}$ matrix are then sorted out for all geometries using the orbital diabatization procedure outlined above and subsequently the AO to MO transformation of the integrals is performed using the standard expressions

$$
\begin{aligned}
\langle i|\hat{h}| j\rangle_{[R]} & =\sum_{\mu \nu} C_{\mu i,[R]}^{*} C_{\nu j,[R]}\langle\mu|\hat{h}| \nu\rangle_{[R]} \\
\langle i j|\hat{v}| k l\rangle_{[R]} & =\sum_{\mu \nu \lambda \sigma} C_{\mu i,[R]}^{*} C_{\nu j,[R]}^{*} C_{\lambda k,[R]} C_{\sigma l,[R]}\langle\mu \nu|\hat{v}| \lambda \sigma\rangle_{[R]}
\end{aligned}
$$

where $\mu, \nu, \lambda, \sigma$ are the $\mathrm{AO}$ and $i, j, k, l$ are the $\mathrm{MO}$ indices. In the N-SQR calculations we neglect the NAC terms related to the orbital basis, Eqs. (48) and (49), and show later when comparing to non-adiabatic first quantization calculations including the full $a b$ initio couplings that this approximation is an excellent one in the present case.

Three different representations of the electronic primitive basis of $\mathrm{HeH}^{+}$are considered and compared. These are termed S-DOF, FS-DOF(I) and FS-DOF(II), and their tree representations are illustrated in Fig. 2. On the one hand we use a S-DOF representation with three electronic modes formed by combining the alpha and beta spin orbitals of $(1,2),(3)$ and $(4,5)$ spatial MOs (see Fig. 2a). The other extreme is FS-DOF(II), where all electronic degrees of freedom are combined into a single FS-DOF. In the latter case, due to the presence of two electrons of opposite spin in the system, the underlying Fock space with $2^{10}$ configurations shrinks to an FS-DOF with 25 particle-number states and the electronic operators in this space are represented hence by $25 \times 25$ matrices. FS-DOF(I) is an intermediate case in which the

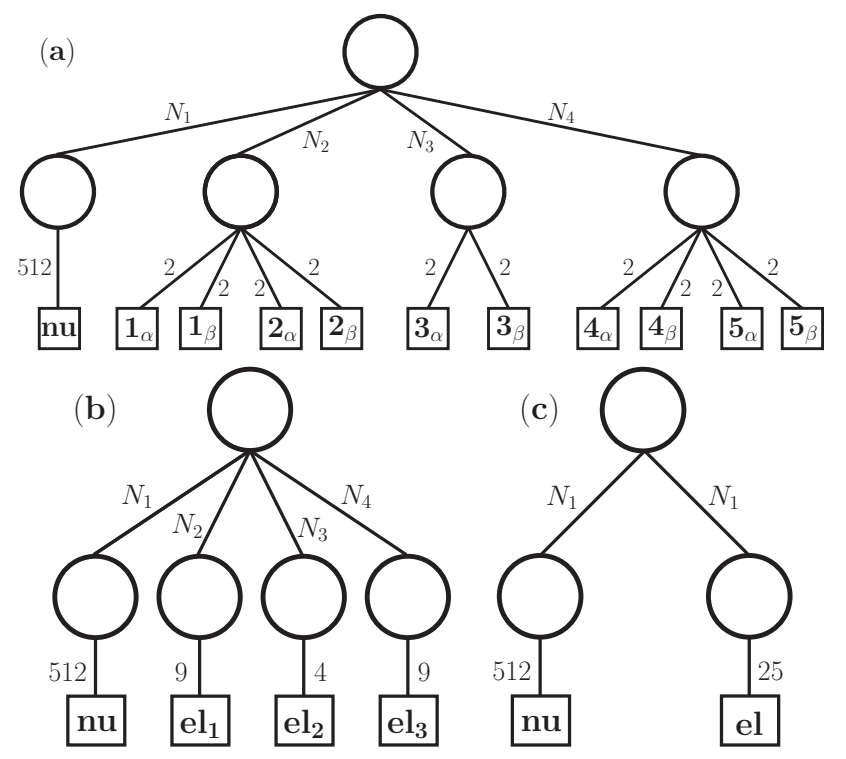

FIG. 2. Tree structures for the MCTDH wavefunctions of the $\mathrm{HeH}^{+}$. (a) MCTDH wavefunction tree, in which spin orbitals are considered as the primitive electronic DOFs (S-DOF). (b) MCTDH wavefunction tree, in which the Fock space (consists of $(4,2,4)$ spin orbitals) is considered as the electronic DOF (FS-DOF(I)) (c) MCTDH wavefunction tree, in which the Fock space (consists of 10 spin orbitals) is considered as the electronic DOF (FS-DOF(II)). "nu" refers to the nuclear DOF and "el $i$ " to the FS-DOF.

S-DOFs in Fig. 2a are collected into FS-DOFs, which become the new electronic primitive degrees of freedom of the MCTDH wavefunction.

The construction of the N-SQR input files for MCTDH, including calls to external electronic structure tools ${ }^{89}$, and the automatic generation of the corresponding input and operator files, is performed by dedicated modules written in Python, which will be made available in a future release of the Heidelberg MCTDH program package $^{72}$. The wavepacket propagation in both the traditional and N-SQR calculations is done as well using the Heidelberg MCTDH program suite ${ }^{72}$.

\section{RESULTS AND DISCUSSION}

\section{A. Electronic eigenenergies from SQR calculations}

First of all, we apply the $a b$ initio MCTDH-SQR machinery to retrieve the correct adiabatic electronic energies, the potential energy curves, from the electronic SQR Hamiltonian at fixed nuclear geometries. In the first quantization calculations, the adiabatic electronic energies for the lowest five ${ }^{1} \Sigma^{+}$states of $\mathrm{HeH}^{+}$are obtained 


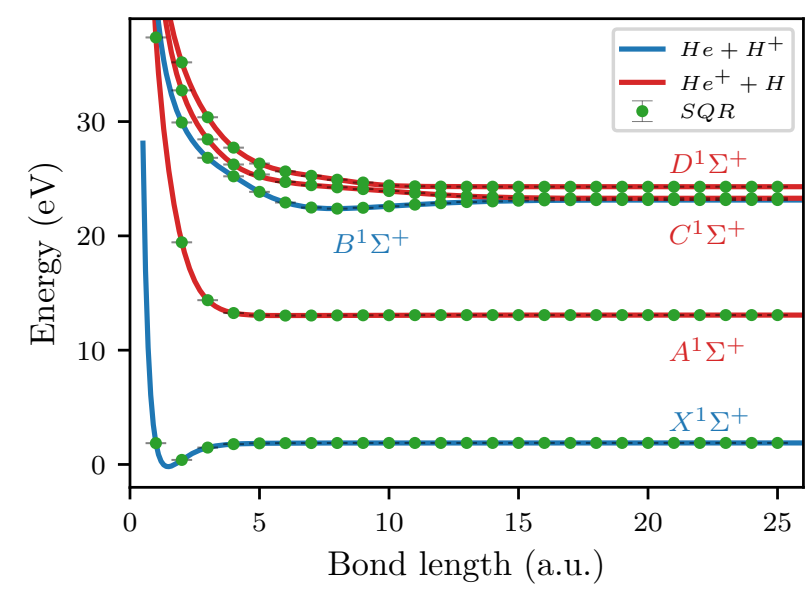

FIG. 3. Comparison of first quantization full configuration interaction and second quantization representation potential energy surfaces.

through diagonalization of the full configuration interaction (FCI) Hamiltonian in the space of the 10 lowest energy HF spin-orbitals of $\Sigma$ symmetry, and are shown as solid lines in Fig. 3. The calculations of the potential energy curves were performed with the MOLPRO package $^{90}$. In SQR, the PECs are obtained through time propagation of the MCTDH-SQR wavefunction

$$
\begin{aligned}
|\Psi(t=0)\rangle & =\left|1_{\alpha}, 1_{\beta}\right\rangle \\
& +\left|1_{\alpha}, 2_{\beta}\right\rangle-\left|1_{\beta}, 2_{\alpha}\right\rangle \\
& +\left|1_{\alpha}, 3_{\beta},\right\rangle-\left|1_{\beta}, 3_{\alpha}\right\rangle \\
& +\left|1_{\alpha}, 4_{\beta}\right\rangle-\left|1_{\beta}, 4_{\alpha}\right\rangle \\
& +\left|1_{\alpha}, 5_{\beta}\right\rangle-\left|1_{\beta}, 5_{\alpha}\right\rangle .
\end{aligned}
$$

in the same spin-orbital space. The electronic wavefunction is represented with either one or three FS-DOFs (electronic part of the Figs. 2b and c, respectively) and the performed MCTDH-SQR calculations are numerically exact. The wavefunction (59) is spin-singlet and overlaps with all five ${ }^{1} \Sigma^{+}$states under consideration. The electronic eigenenergies are obtained from the maxima of the peaks in the power spectrum obtained from the Fourier transform of the autocorrelation function

$$
\sigma(E)=\frac{1}{\pi} \operatorname{Re} \int_{0}^{\infty} e^{i E t}\langle\Psi \mid \Psi(t)\rangle d t .
$$

The SQR electronic eigenenergies are shown as green marks superimposed in Fig. 3. The purpose of this comparison is to illustrate the correctness of our implementation and to hint at the possibility of computing $a b$ initio electronic energies and spectra in an SQR setting through time propagation. For example, an $a b i n i-$ tio time-dependent SQR propagation may serve to obtain ionization electronic spectra, and to calculate the electronic dynamics of molecular systems with a strong multi-configurational character, i.e. static correlation. Applications in these areas remain to be explored.

\section{B. Non-adiabatic dynamics in the N-SQR framework}

\section{Comparison between FQBH and N-SQR}

Upon photoexcitation, the $\mathrm{HeH}^{+}$molecule can dissociate into two main fragmentation channels: $\mathrm{He}+\mathrm{H}^{+}$and $\mathrm{He}^{+}+\mathrm{H}$. The five lowest adiabatic ${ }^{1} \Sigma^{+}$potential energy curves are shown in Fig. 3 for comparison, where blue and red colors indicate the $\mathrm{He}+\mathrm{H}^{+}$and $\mathrm{He}^{+}+\mathrm{H}$ channels, respectively.

The photodissociation cross section of $\mathrm{HeH}^{+}$is obtained both in the FQBH and N-SQR approaches using a complex absorbing potential (CAP) of the form $W(R)=-i \eta\left(R-R_{c}\right)^{2}$ (for $R>R_{c}$ ) with $R_{c}=20 a_{0}$, $\eta=1.935 \cdot 10^{-3}$, and perfoming a flux analysis of the propagated wavepacket ${ }^{91}$. For the comparison in this work we consider only the case that the light is polarized along the molecular axis. The propagated wavepacket is generated by application of the $\hat{M}^{(z)}$ dipole operator to the vibrational and electronic ground state of the system. In the FQBH setting this operator reads

$$
\hat{M}^{(z)}(R)=\sum_{s} \mu_{0 s}^{(z)}(R)(|0\rangle\langle s|+| s\rangle\langle 0|),
$$

where $s$ refers to excited adiabatic electronic states and $\mu_{0 s}(R)$ is the corresponding transition-dipole matrix element. In the N-SQR setting the dipole operator reads

$$
\hat{M}^{(z)}(R)=\sum_{i j}\left\langle\varphi_{i}(R)|\hat{\mu}| \varphi_{j}(R)\right\rangle \hat{b}_{i}^{\dagger} \hat{b}_{j}
$$

which is a one-body operator and the MO dipole integrals are obtained using the integral engine of the PSI4 electronic structure package ${ }^{89}$.

In the FQBH calculation, the fragmentation channelresolved cross sections can be obtained by projecting, before the flux analysis, the propagated wavefunction onto each electronic state and subsequently summing up the cross sections of the $X^{1} \Sigma^{+}$and $B^{1} \Sigma^{+}$states for the $\mathrm{He}+\mathrm{H}^{+}$channel, and the cross sections of the $A^{1} \Sigma^{+}$, $C^{1} \Sigma^{+}$and $D^{1} \Sigma^{+}$states for the $\mathrm{He}^{+}+\mathrm{H}$ channel. In the $\mathrm{N}-\mathrm{SQR}$ representation there are no electronic states to help us resolve the two fragmentation channels. Nonetheless, they can be separated by projecting the wavefunction onto the occupation-number sub-space in which all $\mathrm{H}$-atom spin-orbitals are empty with the projector

$$
\hat{\mathcal{P}}_{H^{+}}=\prod_{i}\left(1-\hat{n}_{i_{H}}\right)
$$

where $i_{H}$ is the index of the molecular spin-orbitals with pure $H$ character. These are well defined in the region of the CAP. This projector singles out the part of the wavefunction dissociating into the $\mathrm{He}+\mathrm{H}^{+}$channel, 

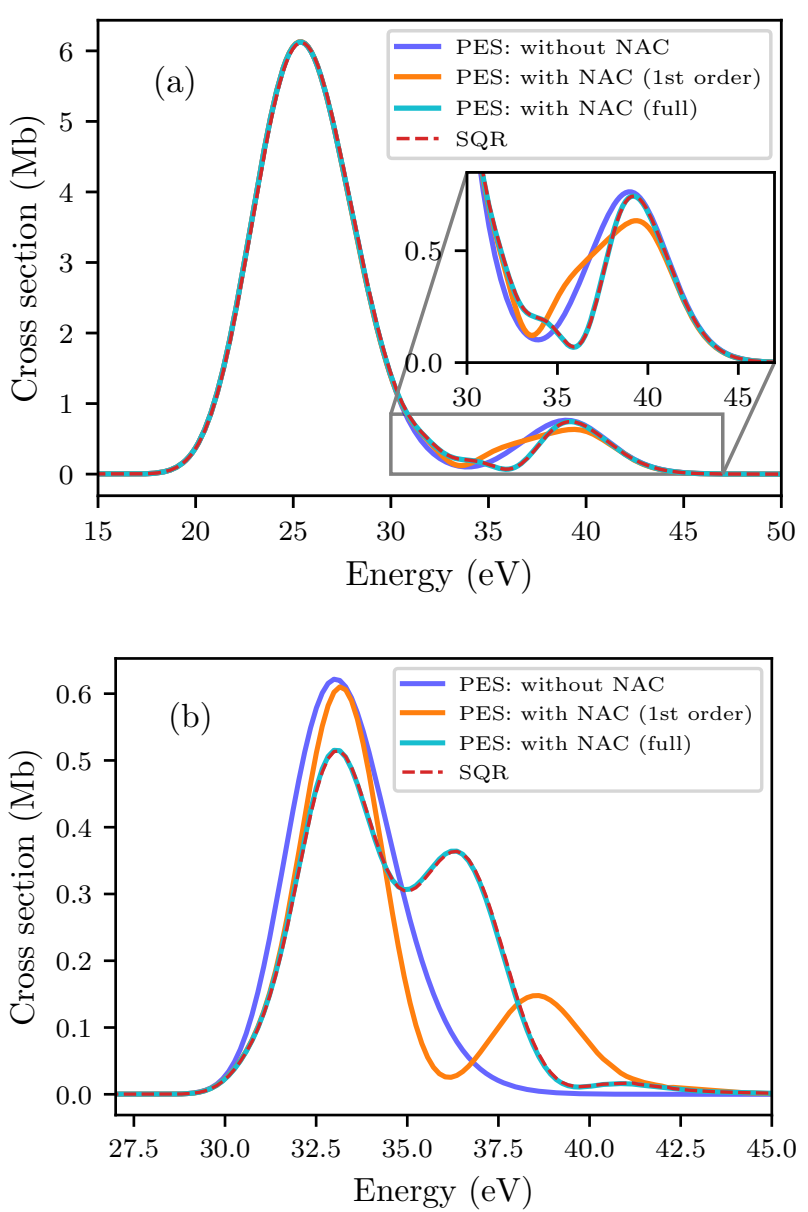

FIG. 4. Photodisssociation cross section into (a) the $\mathrm{He}^{+}+\mathrm{H}$ and (b) the $\mathrm{He}+\mathrm{H}^{+}$channels.

and $\hat{\mathcal{P}}_{H}=1-\hat{\mathcal{P}}_{H^{+}}$projects onto the remaining channel, $\mathrm{He}^{+}+\mathrm{H}$.

Fig. 4a shows the photodissociation cross section of $\mathrm{HeH}^{+}$into $\mathrm{He}^{+}+\mathrm{H}$. In the FQBH description the largest peak centered at about $25 \mathrm{eV}$ originates from dissociation in the $A^{1} \Sigma^{+}$state. This state is energetically far from the next group of excited states and there is negligible participation of it in non-adiabatic population transfer between the dissociation channels. The cross section in the region of this peak is identical for the FQBH calculations with and without NAC, and N-SQR.

On the other hand, the peak centered between 38 and $40 \mathrm{eV}$ arises due to the dissociation through $C^{1} \Sigma^{+}$and $D^{1} \Sigma^{+}$electronic states, which feature non-adiabatic couplings between them and with the $B^{1} \Sigma^{+}$electronic state. The inset in Fig. 4a illustrates how the cross section in this energy region is neither correctly described by an adiabatic description without NACs nor by the inclusion of only first order NAC terms ${ }^{92}$. The FQBH with full NAC and the N-SQR calculation (fully converged) agree exactly. The importance of the second order NAC terms
$\left(D_{p q}^{\alpha}\right.$ in Eq. B12) is also highlighted here, as the calculation without the second order contribution cannot reproduce the shape and the total area of the spectrum.

The non-adiabatic effects in the photodissociation cross section of channel $\mathrm{He}+\mathrm{H}^{+}$, shown Fig. 4b, are even more pronunced. The adiabatic FQBH calculation presents a single peak at $38 \mathrm{eV}$, which correlates with population initially transferred to the $B^{1} \Sigma^{+}$electronic state by the photoabsorption process. A second peak at 36 to $38 \mathrm{eV}$ appears due to the non-adiabatic transitions from the $C^{1} \Sigma^{+}$and $D^{1} \Sigma^{+}$states to the $B^{1} \Sigma^{+}$electronic state as the molecule dissociates. Again, the calculation with only first order NAC terms ${ }^{92}$ does not reproduce the spectrum correctly; the second peak appears too high in energy and the intensity and the area are not correct, highlighting again the importance of the second order terms. On the other hand, the N-SQR calculation agrees exactly with the FQBH calculation with the full first and second order NACs. This means, the non-adiabatic transitions and interference effects between the photodissociation channels are correctly captured within the N-SQR formulation. Moreover, one sees how, in this particlar example, the simple diabatization of the molecular orbitals based on rearranging them to guarantee the continuity of the corresponding matrix elements is a very good approximation. All relevant non-adiabatic effects are captured by the propagated N-SQR wavefunction. We emphasize again, that we could neglect the non-adiabatic coupling terms Eqs. (47-49) in the N-SQR calculations. The $R$ dependence of the orbitals is rather weak, whereas the $R$ dependence of the adiabatic state functions can be very strong.

\section{Convergence and numerical aspects of N-SQR}

Figure 5 compares the photodissociation cross section for the numerically converged S-DOF, FS-DOF(I) and FS-DOF(II) calculations and for the two dissociation channels. Table I compares the number of SPFs, number of Hamiltonian terms and CPU time of the three different calculations, for which the corresponding wavefunction trees are shown in Fig. 2. When brought to numerical convergence, the various representations yield, as expected, identical results. Next, we focus on the FS-DOF(II) calculations and study their convergence behaviour. When the 10 spin-orbitals corresponding to a Fock-space of 1024 states are combined and one uses that only one spin- $\alpha$ and one spin- $\beta$ electrons is present, this number reduces to $2 \times\left(\begin{array}{l}5 \\ 1\end{array}\right)=25$ possible primitive states for the electrons with total spin projection onto the $z$-axis $S_{z}=0$. The nuclear DOF ( $R$-grid) consists of 512 grid points and spans the range $(0.5,30.0)$ au. The photodissociation cross section of $\mathrm{HeH}^{+}$into $\mathrm{He}+\mathrm{H}^{+}$channel is shown in Fig. 6 for an increasing number of SPFs for the electronic and nuclear modes, which are chosen equal to avoid redundant configurations. For the Hartree product case $(\mathrm{SPF}=1)$, the cross section features only one peak, 

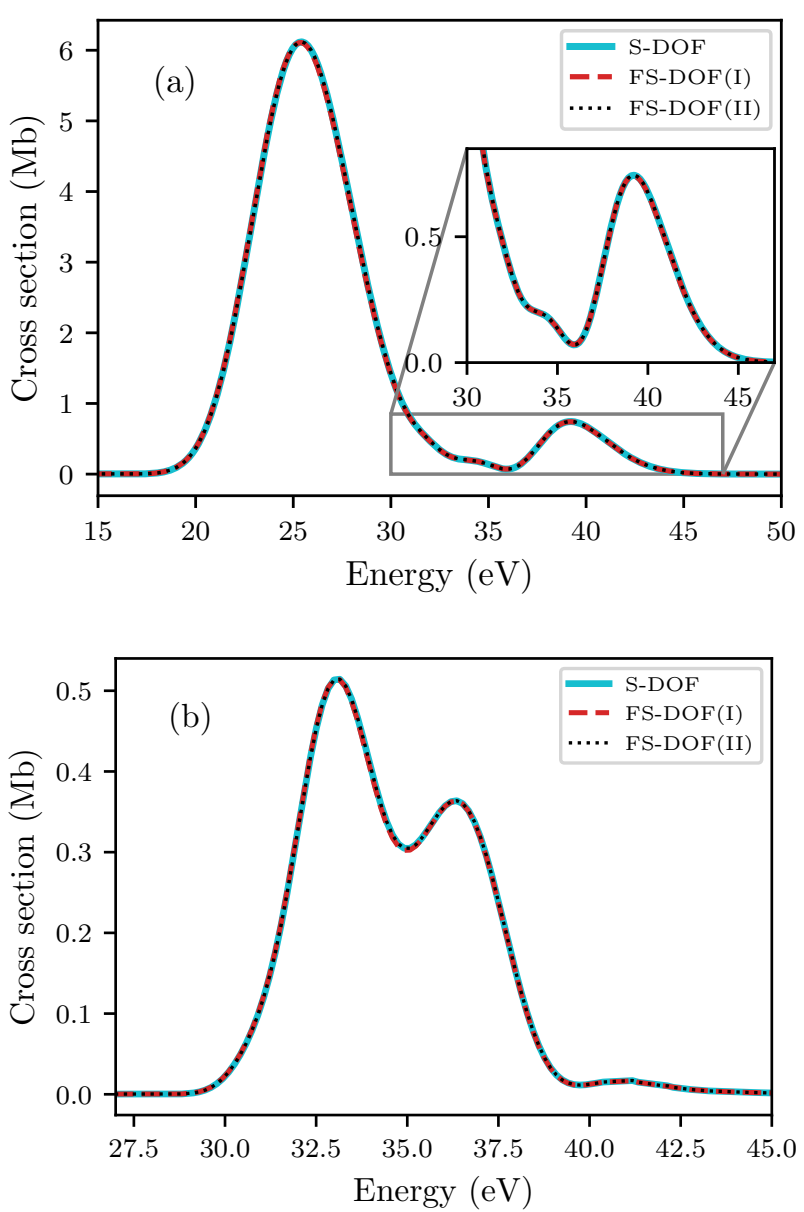

FIG. 5. Photodissociation cross section of $\mathrm{HeH}^{+}$using the SDOF and FS-DOF representations. (a) $\mathrm{He}^{+}+\mathrm{H}$ channel. (b) $\mathrm{He}+\mathrm{H}^{+}$channel

which is centered in the wrong energy region. This is because of the complete neglect of correlation between electronic and nuclear DOF. Note that, since in this example the electronic spin-orbitals are fully combined into a single FS-DOF, the only approximation in the total wavefunction is the missing electron-nuclear correlation. The electronic correlation is treated, within the FS-DOF, exactly. The addition of a second SPF already brings the photodissociation cross section to the correct energy region centered around $35 \mathrm{eV}$. $6 \mathrm{SPF}$ provide already a qualitatively correct description, with a double-shoulder structure, and 8 SPfs and above yield the numerically converged result.

It is interesting to note that, within the 25 primitive states with $S_{z}=0$ in the FS-DOF it is possible to form 15 spin-singlet and 10 spin-triplet linear combinations, or spin-adapted configurations in the language of quantum chemistry. Therefore, a numerically exact calculation of the photodissociation cross section in the spin-singlet manifold requires 15 SPFs. Convergence of
TABLE I. Number of Hamiltonian operator terms and wallclock time for three N-SQR calculations. The S-DOF and FSDOF calculations use the tree in Fig. 2. The calculations have been performed with 16 CPUs using shared-memory parallelization on the same machine and CPU type, namely, DualCore Intel Xeon, processor type E5-2650 v2 running at 2.6 $\mathrm{GHz}$ and the wall-clock times are intended for their relative comparison only.

\begin{tabular}{lccr}
\hline \hline DOF repr. & Hamil. terms & SPFs & Wall time (h:m) \\
\hline S-DOF & 2100 & $(15,9,4,9)$ & $64: 18$ \\
FS-DOF(I) & 1812 & $(15,9,4,9)$ & $45: 25$ \\
FS-DOF(II) & 1300 & $(15,15)$ & $11: 15$ \\
FS-DOF(II) & 1300 & $(10,10)$ & $1: 01$ \\
FS-DOF(II) & 1300 & $(8,8)$ & $0: 15$ \\
\hline \hline
\end{tabular}

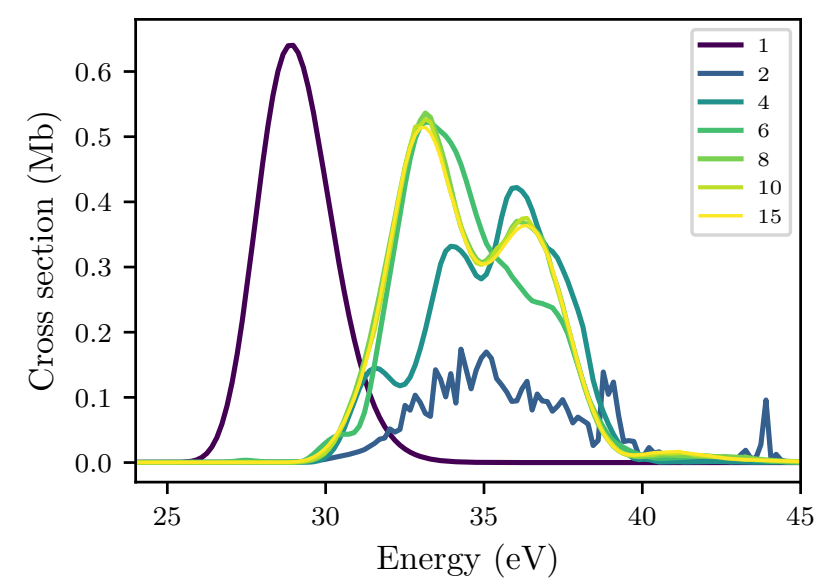

FIG. 6. Photodissociation cross section of $\mathrm{HeH}^{+}$into $\mathrm{He}+\mathrm{H}^{+}$ channel with different number of SPFs

the MCTDH-SQR wavefunction is reached for a smaller number of SPFs because some of the primitive states correspond to occupation patterns of the spin-orbitals with a very high energy and consequently a negligibly small population.

Finally, we discuss the convergence of the photodissociation cross section with of the $\mathrm{He}+\mathrm{H}^{+}$fragmentation channel as a function of the cutoff value for the Hamiltonian terms. The costs of an MCTDH calculation scale roughly linearly with the number of Hamiltonian terms, and therefore it is important to keep this number as low as possible. As was mentioned previously, we vary only the cutoff value for the 3 - and 4 -index terms of the twoelectron part of the electronic Hamiltonian, whereas the 2-index terms (Coulomb and exchange integrals) and the one-electron terms have in all cases a much smaller cutoff of $10^{-9} \mathrm{au}$, which is standard in most MCTDH calculations $^{72}$. The cutoff values are given in Table II. The maximum number of terms, 1300, refers to the FS-DOF 


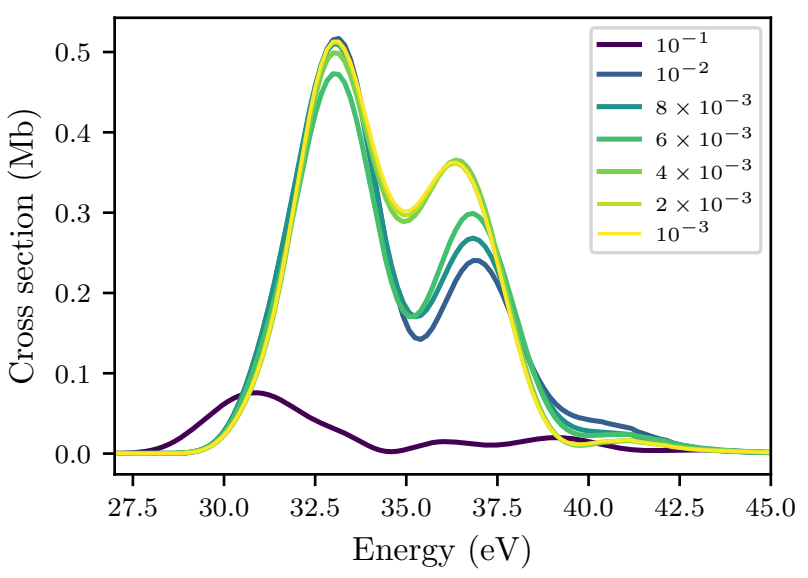

FIG. 7. Comparison of photodissociation cross section of $\mathrm{HeH}^{+}$into $\mathrm{He}+\mathrm{H}^{+}$channel with different cutoff for the 3and 4- index terms of the Hamiltonian. Cutoff for 2-index terms is $10^{-9}$ a.u.

TABLE II. Number of Hamiltonian terms with different cutoff values for the 3- and 4- index terms of the Hamiltonian

\begin{tabular}{lr}
\hline \hline Cutoff & Number of Hamiltonian terms \\
\hline $10^{-1}$ & 140 \\
$10^{-2}$ & 876 \\
$8 \cdot 10^{-3}$ & 956 \\
$6 \cdot 10^{-3}$ & 1068 \\
$4 \cdot 10^{-3}$ & 1204 \\
$2 \cdot 10^{-3}$ & 1284 \\
$10^{-3}$ & 1300 \\
$10^{-9}$ & 1300 \\
\hline \hline
\end{tabular}

setting, which is already smaller than the maximum number of terms of the equivalent S-DOF calculation, 2100. A cutoff of $10^{-1}$ results in the cross section being qualitatively wrong, as seen in Fig. 7. However, the features are in the right energy region because the energy of the diagonal matrix elements between Fock states of the N-SQR Hamiltonian are not affected by the cutoff. A cutoff of $10^{-2}$ with roughly $70 \%$ of the uncut Hamiltonian terms results in a qualitatively correct photodissociation, and convergence is reached with $4 \cdot 10^{-3}$ au, which includes about $80 \%$ of the Hamiltonian terms. Although cutting Hamiltonian terms is not very helpful in the present case, it remains to be benchmarked in more detail in future works on larger molecules.

\section{Properties of the N-SQR approach}

The N-SQR approach has some limitations. A major drawback is the large number of Hamiltonian terms resulting from the second-quantized description of the electrons, which grows proportionally to $M^{4}$, where $M$ is the number of spin-orbitals used to describe the electronic Fock space. This growth can be mitigated by introducing a cutoff for three- and four-index electronic integrals, by freezing orbital occupations, or by pruning configurations from FS-DOFs. (cf. Tab. I). For example, we have pruned the FS-DOFs by removing occupation basis states that are incompatible with the total number of $\alpha$ and $\beta$ electrons of the system. The FS-DOF representation has the added advantage over S-DOFs that the resulting operator terms acting on the FS-DOFs are extremely sparse. This opens the possibility towards avoiding explicit matrix-vector multiplications in their application to the wavefunction.

The other major drawback of the approach is the fact that the matrix elements of the second-quantized electronic Hamiltonian, the one- and two-body integrals $h_{i j}(\boldsymbol{R})$ and $v_{i j k l}(\boldsymbol{R})$, become nuclear-space potential operators, in general of dimensionality $3 N-6(3 N-5$ for linear systems), where $N$ is the number of atoms in the system. We have limited this first application of the NSQR approach to a diatomic system, where the electronic integrals are 1-dimensional functions of the internuclear distance. Although applications to multidimensional systems have not been undertaken yet, there are grounds to believe that this will be feasible. One- and two-body integrals have a much simpler dependency on the nuclear coordinates than potential energy surfaces, and they can possibly be fit with simple functional forms and few parameters each. It may also be possible to expand the electronic integrals around a reference geometry up to, e.g., second order, thus yielding the equivalent of a vibronic coupling Hamiltonian in an N-SQR representation.

A major feature of the N-SQR approach is that it circumvents the construction of potential energy surfaces before the quantum dynamics simulation, and hence it is not based on a group Born-Oppenheimer approxima$\operatorname{tion}^{2,4}$. This can be advantageous in situations where the number of relevant electronic states that would have to be pre-calculated becomes very large, thus complicating the quantum chemistry and diabatization procedures involved. Possible applications include, for example, reactions involving metal complexes, in which nonadiabatic effects are intermixed with the strong multiconfigurational character of the electronic wavefunction ${ }^{93,94}$. In the photodissociation case we have studied, the observable is the fragmentation channel-resolved cross section. The adiabatic electronic states and potentials are not directly related to these quantities and are not observable in experiments that would determine the yield of each charged fragment only. In FQBH calculations, PES and NACs play often an auxiliary role to reach the actual measurable quantities. 
However, even though the group Born-Oppenheimer approximation is circumvented in N-SQR, the approach does not treat nuclei and electrons on the same footing ${ }^{52,53}$. The latter results in large couplings between the positions of the nuclei and the electrons due to their Coulomb attraction. Instead, the electrons appear just through the occupation of the spin-orbitals, from which only pre-computed one- and two-body integrals are needed. The underlying basis of molecular spin-orbitals can be generated through some previous first-quantization mean-field calculation and then properly diabatized, like in this work, or by some other suitable position-dependent transformation of atomic orbitals. The introduction of the electrons via the matrix elements of single-particle states instead of via the matrix elements of all-electron states (i.e. PES, NAC) provides great flexibility and, crucially, it removes the trivial "electrons-follow-the-nuclei" correlation similarly as in the Born-Oppenheimer approximation.

A clear advantage of circumventing the introduction of many-body electronic states is that only the molecular spin-orbitals need to be diabatized, which is a much simpler task than diabatizing electronic states ${ }^{18,20,83-86}$. Alternatively, the corresponding orbital non-adiabatic couplings in Hamiltonian (47) may be computed. As we have seen in the $\mathrm{HeH}^{+}$example, once diabatic orbitals and their matrix elements are introduced, the non-adiabatic dynamics is fully captured within the time-evolution of the nuclear-electronic wavepacket.

\section{SUMMARY AND CONCLUSIONS}

In this work we introduce an $a b$ initio quantum mechanical approach to treat the non-adiabatic quantum dynamics of electrons and nuclei based on a first quantization representation of the nuclei and a second quantization representation (SQR) of the electronic subsystem that overcomes the calculation of potential energy surfaces. We derive the full non-relativistic molecular Hamiltonian in this mixed representation, the N-SQR Hamiltonian (47), in which all degrees of freedom, nuclear coordinates and electronic spin-orbital occupations, are distinguishable, thus making the wavefunction representation amenable to the multi-configurational timedependent Hartree (MCTDH) ansatz and to its multilayer generalization, i.e. to a hierarchical Tucker tensor decomposition. Possibly, other related tensor contraction approaches can also be used. In this work we describe the application of the N-SQR Hamiltonian in conjunction with the MCTDH-SQR method for the time-propagation of the wavefunction, which, for fermions, is based on a Jordan-Wigner transformation of the fermionic operators to ladder spin- $1 / 2$ and auxiliary sign-change operators.

After describing the general aspects of the MCTDHSQR approach for fermions, we introduce the concept of Fock-space degrees of freedom (FS-DOF), which group together several spin degrees of freedom into a sub-Fock space. This is shown to have several advantages: unneeded primitive occupation states can be pruned from the FS-DOF, which automatically reduces the number of Hamiltonian terms in the SQR. Operator terms acting on the FS-DOFs are very sparse, thus making their application more efficient. In this paper's application we have grouped up to 10 spin-orbitals in an FS-DOF, which results in 1024 internal states of the primitive degree of freedom before pruning, and larger FS-DOF can be handled by our code.

The N-SQR approach requires either diabatic orbitals or the calculation of the orbital non-adiabatic couplings (NACs) in Hamiltonian (47). Here we have used a simple diabatization procedure based on the rearrangement of the molecular orbitals for diatomic systems and eventual phase corrections. With these orbitals, we obtain a perfect agreement between N-SQR and traditional first quantization Born-Huang (FQBH) calculations based on adiabatic electronic eigenstates and NAC elements between them. We also describe how to choose the initial SPFs for the corresponding MCTDH calculations, which in the context of MCTDH-SQR is, as recently pointed out by Manthe, of utmost importance.

We apply the N-SQR approach to the photodissociation cross section of the $\mathrm{HeH}^{+}$molecule in the extreme ultraviolet photon energy range and focus on the parallel polarization case $\left(X^{1} \Sigma \rightarrow^{1} \Sigma\right.$ transitions), which has been studied theoretically ${ }^{87,95}$ and experimentally (at the single photon energy of $38.7 \mathrm{eV})^{96}$ before. $\mathrm{HeH}^{+}$fragments into either $\mathrm{He}+\mathrm{H}^{+}$or $\mathrm{He}^{+}+\mathrm{H}$. The fragmentation channel-resolved photodissociation cross section in the energy region between 35 and $40 \mathrm{eV}$ presents interference structures that originate from the electron transfer between the two atoms as they are starting to separate. These effects are particularly pronounced in the $\mathrm{He}+\mathrm{H}^{+}$channel, where the highest energy peak of the cross section is absent in an adiabatic picture. There is complete agreement between the fully converged N-SQR and FQBH calculations including the full electronic state NACs up to second order in the adiabatic representation. Neglecting the second order NACs in the FQBH calculations does not lead either to the correct cross sections.

We also study the accuracy of the N-SQR calculation as a function of the number of SPFs used in the MCTDH wavefunction. The calculations improve monotonically to the exact result with the number of SPFs, and by changing the number of SPFs of the various degrees of freedom the amount of correlation between nuclei and electrons, and between different sub-Fock spaces of the electronic system, can be controlled. Presently, we have implemented the mapping strategy for the application of mode operators to FS-DOF degrees of freedom within the Heidelberg MCTDH package. We still see room for improvements in the integration of the (ML-)MCTDH equations of motion in N-SQR applications. We emphasize that we are introducing the formalism and a proofof-concept application, and that emphasis has not been put yet on computational performance. 
The main drawbacks of the ab initio N-SQR approach have been presented and include the scaling of the number of Hamiltonian terms in the SQR with the 4th power of the number of spin-orbitals. We have discussed how these drawbacks can be, in part, mitigated. On the other hand, N-SQR circumvents the introduction of either adiabatic or diabatic electronic states, which can be of much advantage in situations where the number of electronic states that need to be considered in non-adiabatic processes is large and their energy spacings are commensurate with typical separations between vibrational energy levels. However, it is important to recognize that the NSQR strategy to treat the coupled nuclear and electronic dynamics in molecules is not a silver bullet. Situations in which the quantum dynamics can be captured within a window of few electronic states will, in general, not benefit from the approach. Through the selected example we illustrate the salient features of the method, hint at possible application domains, and lay down the theoretical and practical foundations for subsequent developments and approximations based on the N-SQR formalism. The actual range of applications where the approach will prove to be useful remains an open question to be explored in future work.

\section{SUPPLEMENTARY MATERIAL}

See the supplementary material for the tabular data of the NACME, dipole moment and transition dipole moments among the ground and excited electronic states of $\mathrm{HeH}^{+}$.

\section{DATA AVAILABILITY}

The data that support the findings of this study are available in tabular form in the supplementary materials.

\section{ACKNOWLEDGMENTS}

We thank Prof. H.-D. Meyer for his comments on the manuscript and important assistance with the MCTDH calculations. The authors declare no conflicts of interest.

\footnotetext{
${ }^{1}$ M. Born and J. R. Oppenheimer, Ann. Physik 84, 457 (1927).

${ }^{2}$ M. Born and K. Huang, Dynamical Theory of Crystal Lattices, 5th ed., edited by D. H. Wilkinson and W. Marshall, The International Series of Monographs on Physics (Oxford University Press, Ely House, London, 1968).

${ }^{3}$ T. Pacher, L. S. Cederbaum, and H. Köppel, Adv. Chem. Phys. 84, 293 (1993).

${ }^{4}$ G. A. Worth and L. S. Cederbaum, Annu. Rev. Phys. Chem. 55, 127 (2004).

${ }^{5}$ D. Truhlar and C. Mead, Phys. Rev. A 68, 032501 (2003).

${ }^{6}$ W. Domcke, D. R. Yarkony, and H. Köppel, eds., Conical Intersections: Electronic Structure, Dynamics 83 Spectroscopy (World Scientific Pub Co. Inc., 2004).

${ }^{7}$ T. Azumi and K. Matsuzaki, Photochemistry and Photobiology 25, 315 (1977).
}

${ }^{8}$ H. Koeppel, W. Domcke, and L. S. Cederbaum, in Advances in Chemical Physics (John Wiley \& Sons, Inc., 1984) pp. 59-246.

${ }^{9}$ V. Z. P. Isaac B. Bersuker, Vibronic Interactions in Molecules and Crystals (Springer Berlin Heidelberg, 2011).

${ }^{10} \mathrm{~W}$. Domcke, D. R. Yankony, and H. Koeppel, Conical Intersections (World Scientific Publishing Company, 2011).

${ }^{11}$ H. Lischka, M. Dallos, P. G. Szalay, D. R. Yarkony, and R. Shepard, J. Chem. Phys. 120, 7322 (2004).

${ }^{12}$ Z. Li, B. Suo, and W. Liu, J. Chem. Phys. 141, 244105 (2014).

${ }^{13}$ E. C. Alguire, Q. Ou, and J. E. Subotnik, J. Phys. Chem. B 119, 7140 (2014).

${ }^{14}$ I. G. Ryabinkin, J. Nagesh, and A. F. Izmaylov, J. Phys. Chem. Lett. 6, 4200 (2015).

${ }^{15}$ T. Pacher, L. S. Cederbaum, and H. Köppel, J. Chem. Phys. 89, 7367 (1988).

${ }^{16}$ D. R. Yarkony, J. Phys. Chem. A 102, 8073 (1998).

${ }^{17}$ H. Köppel, J. Gronki, and S. Mahapatra, J. Chem. Phys. 115, 2377 (2001)

${ }^{18}$ H. Nakamura and D. G. Truhlar, J. Chem. Phys. 117, 5576 (2002).

${ }^{19}$ J. Jornet-Somoza, B. Lasorne, M. A. Robb, H.-D. Meyer, D. Lauvergnat, and F. Gatti, J. Chem. Phys. 137, 084304 (2012).

${ }^{20}$ K. R. Yang, X. Xu, and D. G. Truhlar, Chem. Phys. Lett. 573, 84 (2013).

${ }^{21}$ M. van Veenendaal, J. Chang, and A. J. Fedro, Phys. Rev. Lett. 104, 067401 (2010).

${ }^{22}$ C. de Graaf and C. Sousa, Chem. Eur. J. 16, 4550 (2010).

${ }^{23}$ Z. Li, M. E. A. Madjet, O. Vendrell, and R. Santra, Faraday Discuss. 171, 457 (2014).

${ }^{24}$ Z. Li and O. Vendrell, Struct. Dyn. 3, 043203 (2016).

${ }^{25}$ T. Renger, V. May, and O. Kühn, Phys. Rep. 343, 137 (2001).

${ }^{26}$ H. Tamura, M. Huix-Rotllant, I. Burghardt, Y. Olivier, and D. Beljonne, Phys. Rev. Lett. 115, 107401 (2015).

${ }^{27}$ S. R. Reddy, P. B. Coto, and M. Thoss, J. Chem. Phys. 151, 044307 (2019).

${ }^{28}$ V. May and O. Kuhn, Charge and energy transfer dynamics in molecular systems (Wiley-VCH John Wiley distributor, Weinheim Chichester, 2004)

${ }^{29}$ M. Schröter, S. Ivanov, J. Schulze, S. Polyutov, Y. Yan, T. Pullerits, and O. Kühn, Phys. Rep. 567, 1 (2015).

${ }^{30}$ P. G. Lisinetskaya and R. Mitrić, Phys. Rev. A 83, 033408 (2011).

${ }^{31}$ J. E. Subotnik, S. Yeganeh, R. J. Cave, and M. A. Ratner, J. Chem. Phys. 129, 244101 (2008).

${ }^{32}$ J. E. Subotnik, A. Jain, B. Landry, A. Petit, W. Ouyang, and N. Bellonzi, Annu. Rev. Phys. Chem. 67, 387 (2016).

${ }^{33}$ A. L. Fetter and J. D. Walecka, Quantum Theory of ManyParticle Systems (Dover Publications Inc., 2003).

${ }^{34} \mathrm{~J}$. Hubbard, Proceedings of the Royal Society of London. Series A. Mathematical and Physical Sciences 276, 238 (1963).

${ }^{35}$ T. Holstein, Ann. Phys. 8, 325 (1959).

${ }^{36}$ C. F. Coll and G. Beni, Solid State Commun. 15, 997 (1974).

${ }^{37}$ L. D. Lathauwer, B. D. Moor, and J. Vandewalle, SIAM Journal on Matrix Analysis and Applications 21, 1253 (2000).

${ }^{38}$ G. Ceruti and C. Lubich, BIT Numerical Mathematics 60, 591 (2020).

${ }^{39}$ H. D. Meyer, U. Manthe, and L. S. Cederbaum, Chem. Phys. Lett. 165, 73 (1990).

${ }^{40}$ U. Manthe, H.-D. Meyer, and L. S. Cederbaum, J. Chem. Phys. 97, 3199 (1992).

${ }^{41}$ M. H. Beck, A. Jäckle, G. A. Worth, and H.-D. Meyer, Phys. Rep. 324, 1 (2000).

${ }^{42}$ H.-D. Meyer and G. A. Worth, Theor. Chem. Acc. 109, 251 (2003).

${ }^{43}$ H.-D. Meyer, F. Gatti, and G. A. Worth, eds., Multidimensional Quantum Dynamics: MCTDH Theory and Applications (WileyVCH, Weinheim, 2009).

${ }^{44}$ H.-D. Meyer, WIREs Comput Mol Sci 2, 351 (2012).

${ }^{45} \mathrm{H}$. Wang and M. Thoss, J. Chem. Phys. 131, 024114 (2009).

${ }^{46} \mathrm{H}$. Wang and M. Thoss, J. Chem. Phys. 138, 134704 (2013).

${ }^{47}$ U. Manthe and T. Weike, J. Chem. Phys. 146, 064117 (2017). 
${ }^{48}$ T. Weike and U. Manthe, J. Chem. Phys. 152, 034101 (2020). ${ }^{49}$ H. Wang and M. Thoss, J. Chem. Phys. 119, 1289 (2003).

${ }^{50}$ U. Manthe, J. Chem. Phys. 128, 164116 (2008).

${ }^{51}$ O. Vendrell and H.-D. Meyer, J. Chem. Phys. 134, 044135 (2011).

${ }^{52}$ D. J. Haxton, K. V. Lawler, and C. W. McCurdy, Phys. Rev. A 83, 063416 (2011).

${ }^{53}$ I. S. Ulusoy and M. Nest, J. Chem. Phys. 136, 054112 (2012).

${ }^{54}$ A. Muolo, E. Mátyus, and M. Reiher, J. Chem. Phys. 149, 184105 (2018).

${ }^{55}$ L. E. Aebersold, I. S. Ulusoy, and A. K. Wilson, Phys. Rev. A 100, 023406 (2019).

${ }^{56}$ J. Caillat, J. Zanghellini, M. Kitzler, O. Koch, W. Kreuzer, and A. Scrinzi, Phys. Rev. A 71, 012712 (2005).

${ }^{57}$ O. E. Alon, A. I. Streltsov, and L. S. Cederbaum, J. Chem. Phys. 127, 154103 (2007).

${ }^{58}$ D. Hochstuhl and M. Bonitz, J. Chem. Phys. 134, 084106 (2011).

${ }^{59}$ T. Sato and K. L. Ishikawa, Phys. Rev. A 88, 023402 (2013).

${ }^{60}$ A. U. Lode, C. Lévêque, L. B. Madsen, A. I. Streltsov, and O. E. Alon, Rev. Mod. Phys. 92, 011001 (2020).

${ }^{61}$ A. Abedi, N. T. Maitra, and E. K. U. Gross, Phys. Rev. Lett. 105, $123002(2010)$.

${ }^{62}$ L. S. Cederbaum, J. Chem. Phys. 138, 224110 (2013).

${ }^{63}$ F. Agostini, A. Abedi, Y. Suzuki, and E. Gross, Mol. Phys. 111, 3625 (2013)

${ }^{64}$ G. Albareda, H. Appel, I. Franco, A. Abedi, and A. Rubio, Physical Review Letters 113, 083003 (2014).

${ }^{65}$ G. Albareda, A. Kelly, and A. Rubio, Physical Review Materials 3, 023803 (2019).

${ }^{66}$ S. K. Min, F. Agostini, I. Tavernelli, and E. K. U. Gross, J. Phys. Chem. Lett. 8, 3048 (2017).

${ }^{67}$ A. Baiardi and M. Reiher, J. Chem. Theory Comput. 15, 3481 (2019).

${ }^{68}$ Q. Meng and H.-D. Meyer, J. Chem. Phys. 138, 014313 (2013).

${ }^{69}$ J. Broeckhove, L. Lathouwers, E. Kesteloot, and P. V. Leuven, Chem. Phys. Lett. 149, 547 (1988).

${ }^{70}$ C. M. Hinz, S. Bauch, and M. Bonitz, J. Phys. Conf. Ser. 696, 012009 (2016).

${ }^{71}$ L. Cao, S. Krönke, O. Vendrell, and P. Schmelcher, J. Chem. Phys. 139, 134103 (2013).

${ }^{72}$ G. A. Worth, M. H. Beck, A. Jäckle, O. Vendrell, and H.-D. Meyer, The MCTDH Package, Version 8.2, (2000). H.-D. Meyer, Version 8.3 (2002), Version 8.4 (2007). O. Vendrell and H.-D. Meyer Version 8.5 (2013). Version 8.5 contains the ML-MCTDH algorithm. Current versions: 8.4.18 and 8.5.11 (2019). Used version: exchange with "Used version" See http://mctdh.unihd.de/.

${ }^{73}$ I. R. Craig, M. Thoss, and H. Wang, J. Chem. Phys. 127, 144503 (2007).

${ }^{74}$ H. Wang and M. Thoss, Chem. Phys. 347, 139 (2008), ultrafast Photoinduced Processes in Polyatomic Molecules - Electronic Structure, Dynamics and Spectroscopy.

${ }^{75}$ M. F. Shibl, J. Schulze, M. J. Al-Marri, and O. Kühn, J. Phys. B: At., Mol. Opt. Phys. 50, 184001 (2017).

${ }^{76}$ S. Zöllner, H.-D. Meyer, and P. Schmelcher, Phys. Rev. A 74, $053612(2006)$.

${ }^{77}$ S. Zöllner, H.-D. Meyer, and P. Schmelcher, Phys. Rev. A 78, 013629 (2008).

${ }^{78}$ O. E. Alon, A. I. Streltsov, and L. S. Cederbaum, Phys. Rev. A 77, 033613 (2008).

${ }^{79}$ P. Jordan and E. Wigner, Zeitschrift für Physik 47, 631 (1928).

${ }^{80}$ Z. Bacic and J. C. Light, J. Chem. Phys. 85, 4594 (1986).

${ }^{81} \mathrm{H}$. Wang and M. Thoss, The Journal of Physical Chemistry A 117, 7431 (2013).

${ }^{82} \mathrm{H}$. Wang and M. Thoss, The Journal of chemical physics 145, 164105 (2016).

${ }^{83}$ T. Pacher, H. Köppel, and L. S. Cederbaum, J. Chem. Phys. 95, 6668 (1991).
${ }^{84}$ W. Domcke and C. Woywod, Chem. Phys. Lett. 216, 362 (1993).

${ }^{85}$ V. Garcia, M. Reguero, R. Caballol, and J. Malrieu, Chem. Phys. Lett. 281, 161 (1997).

${ }^{86}$ H. Nakamura and D. G. Truhlar, J. Chem. Phys. 115, 10353 (2001).

${ }^{87}$ I. Dumitriu and A. Saenz, J. Phys. B: At., Mol. Opt. Phys. 42, 165101 (2009).

${ }^{88}$ T. H. Dunning, Jr., J. Chem. Phys. 90, 1007 (1989).

${ }^{89}$ R. M. Parrish, L. A. Burns, D. G. A. Smith, A. C. Simmonett, A. E. DePrince, E. G. Hohenstein, U. Bozkaya, A. Y. Sokolov, R. D. Remigio, R. M. Richard, J. F. Gonthier, A. M. James, H. R. McAlexander, A. Kumar, M. Saitow, X. Wang, B. P. Pritchard, P. Verma, H. F. Schaefer, K. Patkowski, R. A. King, E. F. Valeev, F. A. Evangelista, J. M. Turney, T. D. Crawford, and C. D. Sherrill, J. Chem. Theory Comput. 13, 3185 (2017).

${ }^{90}$ R. D. Amos, A. Bernhardsson, A. Berning, P. Celani, D. L. Cooper, M. J. O. Deegan, A. J. Dobbyn, F. Eckert, C. Hampel, G. Hetzer, P. J. Knowles, T. Korona, R. Lindh, A. W. Lloyd, S. J. McNicholas, F. R. Manby, W. Meyer, M. E. Mura, A. Nicklass, P. Palmieri, R. Pitzer, G. Rauhut, M. Schütz, U. Schumann, H. Stoll, A. J. Stone, R. Tarroni, T. Thorsteinsson, and H.-J. Werner, "Molpro, a package of ab initio programs designed by H.-J. Werner and P. J. Knowles, version 2002.1," (2002).

${ }^{91}$ A. Jäckle and H.-D. Meyer, J. Chem. Phys. 105, 6778 (1996).

${ }^{92} \mathrm{By}$ first order NAC terms we denote the symmetrized term $\frac{\partial}{\partial R} d_{i j}+d_{i j} \frac{\partial}{\partial R}$. See Appendix B.

${ }^{93}$ C. Bressler, C. Milne, V. Pham, A. ElNahhas, R. M. van der Veen, W. Gawelda, S. Johnson, P. Beaud, D. Grolimund, M. Kaiser, C. N. Borca, G. Ingold, R. Abela, and M. Chergui, Science 323, 489 (2009).

${ }^{94}$ P. Nalbach, A. J. A. Achner, M. Frey, M. Grosser, C. Bressler, and M. Thorwart, J. Chem. Phys. 141, 044304 (2014).

${ }^{95}$ A. Saenz, Phys. Rev. A 67, 033409 (2003).

${ }^{96}$ H. B. Pedersen, S. Altevogt, B. Jordon-Thaden, O. Heber, M. L. Rappaport, D. Schwalm, J. Ullrich, D. Zajfman, R. Treusch, N. Guerassimova, M. Martins, J.-T. Hoeft, M. Wellhöfer, and A. Wolf, Physical Review Letters 98, 223202 (2007).

\section{Appendix A: Born-Huang expansion with the molecular SQR Hamiltonian}

As mentioned in the main text, Hamiltonian (47) is compatible with low-rank tensor decompositions of the wavefunction owing to the distinguishability of the degrees of freedom. Nonetheless, it is illustrative to consider formally a wavefunction expansion analogous to the usual Born-Huang (BH) ansatz, in which all occupationnumber states are explicitly kept:

$$
|\Psi(t)\rangle=\sum_{\{\vec{n}\}} \Phi_{\{\vec{n}\}}(\mathbf{R}, t)|\{\vec{n}\}\rangle
$$

where $|\{\vec{n}\}\rangle$ is the occupation number representation ket of an electronic configuration uniquely described by the set of spin-orbital occupations $\vec{n}$ in terms of the $\psi(\vec{x}, \mathbf{R})$ basis and $\Phi_{\{\vec{n}\}}(\mathbf{R}, t)$ is the corresponding probability amplitude, which, as in the electronic states formulation, is a function of the nuclear positions.

Inserting the ansatz (A1) into the general TDSE while using Hamiltonian (47) results in the TDSE 


$$
\begin{aligned}
i \dot{\Phi}_{\{\vec{m}\}}(\mathbf{R}, t) & =\left[\hat{T}_{n}+\hat{W}_{n}\right] \Phi_{\{\vec{m}\}}(\mathbf{R}, t) \\
& +\sum_{\{\vec{n}\}}\left(\sum_{p q}^{F}\left[h_{p q}(\mathbf{R})-\frac{1}{M} \mathbf{D}_{p q}(\mathbf{R}) \cdot \nabla-\frac{1}{2 M} G_{p q}(\mathbf{R})\right]\left\langle\{\vec{m}\}\left|\hat{b}_{p}^{\dagger} \hat{b}_{q}\right|\{\vec{n}\}\right\rangle\right. \\
& \left.+\frac{1}{2} \sum_{p q r s}^{F} v_{p q r s}(\mathbf{R})\left\langle\{\vec{m}\}\left|\hat{b}_{p}^{\dagger} \hat{b}_{q}^{\dagger} \hat{b}_{r} \hat{b}_{s}\right|\{\vec{n}\}\right\rangle\right) \Phi_{\{\vec{n}\}}(\mathbf{R}, t) .
\end{aligned}
$$

In this BH-SQR formulation of the non-adiabatic dynamics problem, the TDSE contains both differential and potential coupling terms between the nuclear and electronic spaces. If the kinetic coupling terms are kept small or negligible by choosing a quasi-diabatic spin-orbital basis, the remaining non-adiabatic coupling effects are described directly by the time-evolution of the nuclear amplitudes $\Phi_{\{\vec{m}\}}(\mathbf{R}, t)$.

As usual, there is no free lunch and the price to be paid is a potentially much larger space of electronic occupation states as compared to adiabatic electronic states. Therefore, the BH variant of the nuclear-SQR approach, Eq. (A2), may not find much direct use. Conceptually, it is completely equivalent to a first quantization description in which each electronic configuration of the full configuration-interaction matrix carries its own nuclear amplitude. For this reason, practical applications will still be based on the N-SQR formulation of the main text.

\section{Appendix B: Non-adiabatic quantum dynamics in the adiabatic representation}

The electronic part of the total molecular Hamiltonian (29) in the first quantization representation is given as

$$
\hat{H}_{e}=\sum_{i=1}^{N}\left[-\frac{1}{2} \nabla_{i}^{2}-\sum_{\alpha=1}^{M} \frac{Q_{\alpha}}{r_{i \alpha}}+\sum_{j>i} \frac{1}{r_{i j}}\right]
$$

Using the Born-Huang expansion, the total molecular wavefunction is expressed as

$$
\Psi(x, R, t)=\sum_{p} \chi_{p}(x ; R) \Phi_{p}(R, t)
$$

where $\chi_{n}(x ; R)$ are the electronic basis functions that parametrically depend on the nuclear coordinates and $\Phi_{n}(R, t)$ are the nuclear wavefunction at time $t$. Now inserting this wavefunction ansatz into the time dependent Schorödinger equation, one obtains the following coupled equation

$$
i \dot{\Phi}_{q}(R, t)=\hat{T}_{n} \Phi_{q}(R, t)+\sum_{p}\left[V_{p q}(R)-\sum_{\alpha} \frac{1}{2 m_{\alpha}}\left(2 d_{p q}^{\alpha}(R) \frac{\partial}{\partial R_{\alpha}}+G_{p q}(R)\right)\right] \Phi_{p}(R, t)
$$

where

$$
\begin{array}{r}
V_{p q}(R)=\left\langle\chi_{p}(x ; R)\left|\hat{H}_{e}+\hat{W}_{n}\right| \chi_{q}(x ; R)\right\rangle \\
d_{p q}^{\alpha}(R)=\left\langle\chi_{p}(x ; R)\left|\frac{\partial}{\partial R_{\alpha}}\right| \chi_{q}(x ; R)\right\rangle \\
G_{p q}^{\alpha}=\left\langle\chi_{p}(x ; R)\left|\frac{\partial^{2}}{\partial R_{\alpha}^{2}}\right| \chi_{q}(x ; R)\right\rangle
\end{array}
$$

In the adiabatic representation, the electronic basis functions become the eigen functions of the electronic Hamiltonian and thus, the $V$ becomes a diagonal matrix with adiabatic electronic energies in the diagonal. Thus, for one nuclear coordinate, the non-adiabatic coupling operator between $i$ th and $j$ th electronic states is

$$
\Lambda_{i j}=\frac{1}{2 M}\left(2 d_{i j} \frac{\partial}{\partial R}+G_{i j}\right)
$$

Now the first order term itself is not Hermitian. But we can symmetrize it by following

$$
\begin{aligned}
\frac{\partial}{\partial R} d_{i j} & =\frac{\partial}{\partial R}\left\langle\phi_{i}\left|\frac{\partial}{\partial R}\right| \phi_{j}\right\rangle \\
& =\left\langle\frac{\partial \phi_{i}}{\partial R} \mid \frac{\partial \phi_{j}}{\partial R}\right\rangle+\left\langle\phi_{i}\left|\frac{\partial^{2}}{\partial R^{2}}\right| \phi_{j}\right\rangle+\left\langle\phi_{i}\left|\frac{\partial}{\partial R}\right| \phi_{j}\right\rangle \frac{\partial}{\partial R} \\
& =\left\langle\frac{\partial \phi_{i}}{\partial R} \mid \frac{\partial \phi_{j}}{\partial R}\right\rangle+G_{i j}+d_{i j} \frac{\partial}{\partial R} \\
& =\sum_{l}\left\langle\frac{\partial \phi_{i}}{\partial R} \mid \phi_{l}\right\rangle\left\langle\phi_{l} \mid \frac{\partial \phi_{j}}{\partial R}\right\rangle+G_{i j}+d_{i j} \frac{\partial}{\partial R} \\
& =\sum_{l} d_{i l}^{*} d_{l j}+G_{i j}+d_{i j} \frac{\partial}{\partial R}
\end{aligned}
$$


Adding $d_{i j} \frac{\partial}{\partial R}$ to both sides and rearranging

$$
2 d_{i j} \frac{\partial}{\partial R}+G_{i j}=\frac{\partial}{\partial R} d_{i j}+d_{i j} \frac{\partial}{\partial R}-D_{i j}
$$

where

$$
D_{i j}=\sum_{l} d_{i l}^{*} d_{l j}
$$

So, the symmetrized form of the NAC operator reads

$$
\Lambda_{i j}=\frac{1}{2 M}\left(\frac{\partial}{\partial R} d_{i j}+d_{i j} \frac{\partial}{\partial R}-D_{i j}\right) .
$$

Using this symmetrized form of the coupling terms, the Eq. B3 is written as

$$
i \dot{\Phi}_{q}(R, t)=\hat{T}_{n} \Phi_{q}(R, t)+\sum_{p}\left[V_{p q}(R)-\sum_{\alpha} \frac{1}{2 m_{\alpha}}\left(\frac{\partial}{\partial R} d_{p q}^{\alpha}(R)+d_{p q}^{\alpha}(R) \frac{\partial}{\partial R}-D_{p q}^{\alpha}(R)\right)\right] \Phi_{p}(R, t) .
$$

To study the nonadiabatic effects in the photodissociation of $\mathrm{HeH}^{+}$, the uncontracted aug-cc-pVTZ basis is used for both $\mathrm{H}$ and $\mathrm{He}$ atoms. In this work we have considered 10 lowest energy HF spin orbitals of $\Sigma$ symmetry to calculate the potential energy curves, non-adiabatic coupling matrix elements and the transition dipole moments between the lowest $5{ }^{1} \Sigma^{+}$states. Those are given in the supplementary material. 